\title{
Control of an Underactuated Double-Pendulum Overhead Crane using Improved Model Reference Command Shaping: Design, Simulation and Experiment
}

\author{
H. I. Jaafar ${ }^{1}$, Z. Mohamed ${ }^{2 *}$, M. A. Ahmad ${ }^{3}$, N. A. Wahab ${ }^{2}$, L. Ramli ${ }^{4}$ and M. H. Shaheed ${ }^{5}$ \\ ${ }^{1}$ Faculty of Electrical Engineering, Universiti Teknikal Malaysia Melaka, Melaka, Malaysia \\ ${ }^{2}$ School of Electrical Engineering, Universiti Teknologi Malaysia, Johor, Malaysia \\ ${ }^{3}$ Faculty of Electrical and Electronics Engineering, Universiti Malaysia Pahang, Pahang, \\ Malaysia \\ ${ }^{4}$ Faculty of Engineering and Built Environment, Universiti Sains Islam Malaysia, Negeri \\ Sembilan, Malaysia \\ ${ }^{5}$ School of Engineering and Materials Science, Queen Mary University of London, London, \\ UK \\ *Corresponding author: zahar@fke.utm.my, Tel: +607-5557219.
}

\begin{abstract}
This paper presents a new control scheme based on model reference command shaping (MRCS) for an overhead crane, with double-pendulum mechanism effects. The approach has an advantage in achieving an accurate trolley positioning, with low hook and payload oscillations, under various desired trolley positions and parameter uncertainties, without the requirement for measurement or estimation of system parameters. These are challenging in practice. The previously developed MRCS algorithm is improved in order to reduce its design complexity, as well as to ensure that it can be augmented with a feedback controller so that a concurrent controller tuning can be realised. The combined MRCS and feedback controller is used to achieve both, precise trolley positioning, and low hook and payload oscillations. To evaluate the effectiveness and the robustness of the approach, simulations and experiments using a nonlinear model and a laboratory double-pendulum crane are carried out. Under various desired positions and parameter uncertainties that involve varying the cable lengths (payload hoisting) and the payload mass variations, the superiority of the proposed approach is confirmed by achieving higher hook and payload oscillation reductions when compared with a recently proposed feedback controller. In addition, the desired trolley positions are achieved with smoother responses.
\end{abstract}

Keywords: Command shaping; Double-pendulum crane; Hybrid control; Model reference; Particle swarm optimisation 


\section{INTRODUCTION}

As worldwide industrial machine applications, cranes are exclusively used for transferring a variety of massive loads to various locations. In fact, most industrial cranes are considered to be underactuated mechanical systems, which would indicate a lower number of actuators than the degrees of freedom [1]. The control of such an underactuated system is complicated, especially when dealing with double-pendulum mechanism effects [2-3]. For a doublependulum overhead crane (DPOC), the hook and the payload oscillate with different oscillation modes (known as multimode) during transportation, whereby the simultaneous elimination of both oscillations is difficult [4]. This phenomenon becomes extremely challenging under parameter uncertainties, with varying cable lengths (payload hoisting) and payload mass variations that affect the oscillation frequencies, which may lead to positioning errors and significant payload oscillations. These result in a decrease in industrial productivity and they introduce a safety issue [5-7].

Several feedback control approaches have been proposed for various types of crane to enable the cranes to regulate its performance, by ensuring that the actual output is closer to the desired response. These include adaptive control [8], nonlinear control [9-11], sliding mode control [12], time optimal control [13], generalised trajectory modification strategy [14], and fuzzy logic control [15]. For control of the DPOC, feedback control strategies that have been proposed were adaptive [7], linear [16], nonlinear [17-18], and intelligent [19] control approaches. It is known that full state feedback controllers require several sensors for measuring the trolley position, as well as the hook and payload oscillation angles for control action [20]. Nevertheless, the necessary additional sensors require an additional cost, and the payload angle measurements will be difficult, with different payload masses, sizes, and shapes, especially for the double-pendulum mechanism effects [21]. In opposition to the feedback control, a feedforward control has been widely utilised for the oscillation control of a DPOC 
[2,22-23]. A two-mode input shaping technique has been mostly used when effectively applied to the crane [24-26]. Moreover, feedforward shapers, namely, a command shaper [27-28] and a smoother [29-31], with different design approaches have been investigated. Improved input shapers have also been proposed for an overhead crane [32] and a tower crane [33].

Recently, model reference command shaping (MRCS) was proposed by [34] and was designed by using a critically damped reference model for the oscillation control of a DPOC. As an alternative feedforward control, this approach did not require any oscillation feedback sensor. This certainly saves on the cost, especially for multimode systems [35]. Besides, an MRCS has another great advantage when compared to other feedforward control approaches, as prior knowledge of the system's natural frequencies are not required for the design. Therefore, the difficulties for measurements or estimations of the system parameters can be avoided. Simulations and experiments on a DPOC have shown significant hook and payload oscillation reductions under various crane operating conditions [34]. However, this control approach has a major drawback, as it can only be used for oscillation control, and it does not have the ability to precisely drive the trolley to various desired positions. In addition, the design of an MRCS is challenging, for it involves complicated procedures and mathematical formulations.

In order to utilise the advantage that is offered by an MRCS and to achieve an accurate crane positioning, a method to combine it with a feedback controller can be established. However, for a real-time realisation of the combined control structure, a simpler MRCS algorithm, with less mathematical formulations, is required. In the literature, several efforts have been presented for developing a hybrid (combination of feedforward and feedback) control strategy for a DPOC. These include input shaping with a nominal characteristic trajectory [36], a single input fuzzy logic controller [37], and a model reference control [38]. In [39], the input shaping has been augmented with two feedback controllers (i.e. proportional- 
derivative (PD) and proportional (P) controllers) for the control of the trolley position and the deflection of the hook. It has been found that most of the hybrid controllers were designed independently. For the control strategies that involved input/command shaping, they required prior knowledge of either the first frequency mode (i.e. the hook), or the second frequency mode (i.e. the payload), or both of the frequency modes (i.e. the hook and the payload) for the design of the control.

This paper presents an MRCS-based control of an underactuated overhead crane, with double-pendulum mechanism effects, under various desired trolley positions and parameter uncertainties. This involved varying the cable lengths (payload hoisting) and the payload mass variations. In order to retain the great advantage offered by an MRCS and to achieve an accurate crane positioning, the previously developed MRCS scheme was improved, in order to reduce the design complexity and to ensure that it could be augmented with a practical feedback controller. To demonstrate the capability of the proposed control structure, a PID controller was utilised as a practical feedback controller, and the concurrent tuning of the control parameters was realised by using particle swarm optimisation (PSO). The proposed controller is called MRCS-PID, and the main difference as compared to the previous MRCS approach is in term of the control structure. In this case, the MRCS is a feedforward control approach, whereas the proposed MRCS-PID is a combined of feedforward and feedback controllers to achieve a precise trolley positioning and satisfactory oscillation control.

The main contributions of this work when in comparison with the existing body of literature are:

a) To the best of authors' knowledge, this is the first work on designing a hybrid controller (a combined command shaper and feedback controller) for a double-pendulum crane that avoids the requirement for prior knowledge of the system oscillation frequencies. 
b) The hybrid controller was designed, such that a concurrent tuning of the two controllers could be performed, in order to ensure optimal performance in the trolley positioning and the payload oscillation. In addition, the design of the previously developed MRCS algorithm was improved.

Several scenarios of the DPOC that involved the desired trolley positions and the parameter uncertainties were considered. Simulations and experiments using an underactuated nonlinear model and a laboratory DPOC were conducted, so as to investigate the effectiveness of the proposed strategy. The accuracy of the trolley positions and the level of reductions of the hook and payload oscillations were analysed, and these were used to investigate the performance of the controller. Performance comparisons with a recently proposed control algorithm for a DPOC system designed using two PID controllers were also conducted, in order to further verify the robustness of the MRCS-PID control strategy.

\section{MODEL OF A DPOC SYSTEM}

Fig. 1 illustrates the schematic diagrams of a DPOC, without and with the varying cable lengths during the payload hoisting. Distance $x$ indicates the trolley path as it moves from the origin, while $\theta_{1}$ and $\theta_{2}$ represent the hook and the payload oscillation angles, respectively. In addition, $m, m_{1}, m_{2}, l_{1}, l_{2}, g, f_{x}$, and $u$ denote the trolley mass, the hook mass, the payload mass, the hook cable length, the payload cable length, the gravitational constant, the friction coefficient of $x$, and the control force applied on the trolley, respectively. As the work considered for constant and varying cable lengths, dynamic equations of the DPOC under both conditions are presented.

When operating without a varying cable length of $l_{1}$, as illustrated in Fig. 1(a), the dynamic equation of the DPOC can be obtained as [2,23]:

$$
\left(m+m_{1}+m_{2}\right) \ddot{x}+\left(m_{1}+m_{2}\right)\left[l_{1} \ddot{\theta}_{1} \cos \theta_{1}-l_{1} \dot{\theta}_{1}^{2} \sin \theta_{1}\right]
$$




$$
\begin{gathered}
+m_{2} l_{2}\left[\ddot{\theta}_{2} \cos \theta_{2}-\dot{\theta}_{2}{ }^{2} \sin \theta_{2}\right]=u-f_{x} \dot{x} \\
\left(m_{1}+m_{2}\right)\left[l_{1} \ddot{x} \cos \theta_{1}+l_{1}{ }^{2} \ddot{\theta}_{1}+g l_{1} \sin \theta_{1}\right] \\
+m_{2} l_{1} l_{2}\left[\ddot{\theta}_{2} \cos \left(\theta_{1}-\theta_{2}\right)+\dot{\theta}_{2}{ }^{2} \sin \left(\theta_{1}-\theta_{2}\right)\right]=0 \\
m_{2} l_{2}\left[\ddot{x} \cos \theta_{2}+l_{1} \ddot{\theta}_{1} \cos \left(\theta_{1}-\theta_{2}\right)+l_{2} \ddot{\theta}_{2}-l_{1} \dot{\theta}_{1}{ }^{2} \sin \left(\theta_{1}-\theta_{2}\right)+g \sin \theta_{2}\right]=0
\end{gathered}
$$

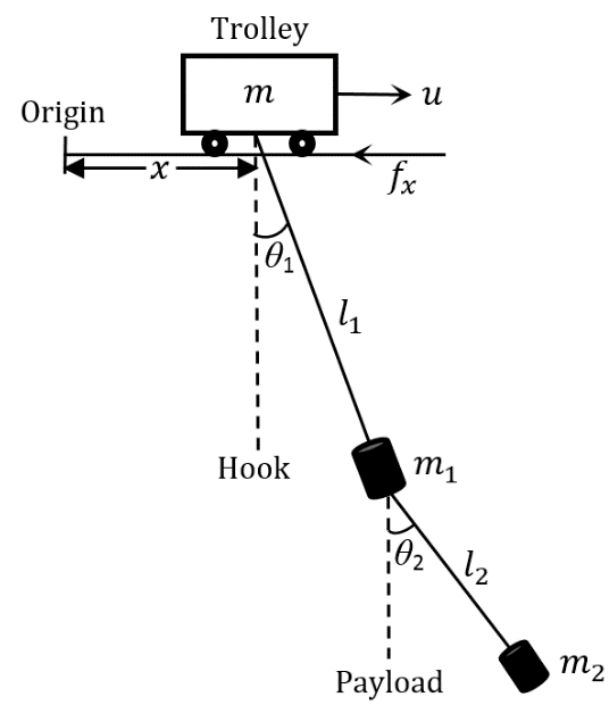

(a)

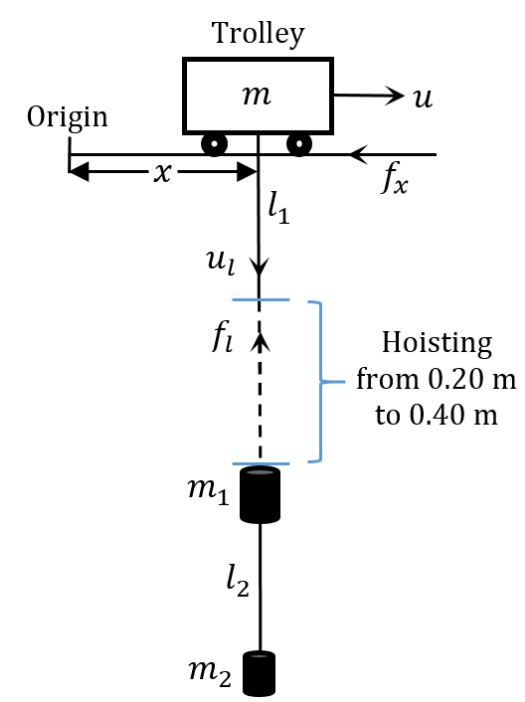

(b)

Fig. 1. A DPOC: (a) Without a varying cable length (b) With a varying cable length

Additionally, the payload was also required to be hoisted (up or down) in practical operations to the desired location. With a varying cable length of $l_{1}$, as illustrated in Fig. 1(b), the dynamic equation of the DPOC can be written as $[7,40]$ :

$$
\begin{gathered}
\left(m+m_{1}+m_{2}\right) \ddot{x}+\left(m_{1}+m_{2}\right)\left[2 \dot{l}_{1} \dot{\theta}_{1} \cos \theta_{1}+l_{1} \ddot{\theta}_{1} \cos \theta_{1}\right. \\
\left.-l_{1} \dot{\theta}_{1}^{2} \sin \theta_{1}+\ddot{l}_{1} \sin \theta_{1}\right]+m_{2} l_{2}\left[\ddot{\theta}_{2} \cos \theta_{2}-\dot{\theta}_{2}^{2} \sin \theta_{2}\right]=u-f_{x} \dot{x} \\
\left(m_{1}+m_{2}\right)\left[\ddot{x}_{1} \cos \theta_{1}+l_{1}^{2} \ddot{\theta}_{1}+g l_{1} \sin \theta_{1}+2 l_{1} \dot{l}_{1} \dot{\theta}_{1}\right] \\
+m_{2} l_{1} l_{2}\left[\ddot{\theta}_{2} \cos \left(\theta_{1}-\theta_{2}\right)+\dot{\theta}_{2}^{2} \sin \left(\theta_{1}-\theta_{2}\right)\right]=0 \\
m_{2} l_{2}\left[\ddot{x} \cos \theta_{2}+l_{2} \ddot{\theta}_{2}+\ddot{l}_{1} \sin \left(\theta_{1}-\theta_{2}\right)-l_{1} \dot{\theta}_{1}{ }^{2} \sin \left(\theta_{1}-\theta_{2}\right)\right. \\
\left.+l_{1} \ddot{\theta}_{1} \cos \left(\theta_{1}-\theta_{2}\right)+2 \dot{l}_{1} \dot{\theta}_{1} \cos \left(\theta_{1}-\theta_{2}\right)+g \sin \theta_{2}\right]=0
\end{gathered}
$$




$$
\begin{gathered}
\left(m_{1}+m_{2}\right)\left[\ddot{x} \sin \theta_{1}+\ddot{l}_{1}-l_{1} \dot{\theta}_{1}{ }^{2}+g\left(1-\cos \theta_{1}\right)\right] \\
+m_{2} l_{2}\left[\ddot{\theta}_{2} \sin \left(\theta_{1}-\theta_{2}\right)-\dot{\theta}_{2}{ }^{2} \cos \left(\theta_{1}-\theta_{2}\right)\right]=u_{l}-f_{l} \dot{l}_{1}
\end{gathered}
$$

where $u_{l}$ and $f_{l}$ denote the hoisting force and friction coefficient of $l_{1}$.

\section{CONTROLLER DESIGNS}

This section presents the improved MRCS algorithm, which can be utilised and augmented with a feedback controller, in order to achieve an accurate trolley positioning, together with higher oscillation reductions of the hook and the payload for an underactuated DPOC. A practical PID feedback controller was used to demonstrate the proposed MRCS-PID control strategy, and a PSO with a new fitness function was introduced for the concurrent tuning of the controller parameters. Using the same PSO algorithm, a recently proposed feedback controller using two PID controllers (PID-PID) was designed and implemented for performance comparisons.

\subsection{MRCS-PID Control Strategy}

Without a requirement for the crane's oscillation frequencies, the MRCS was previously designed as an alternative command shaper by determining an appropriate shaper, $G_{s}(s)$, a

critically damped reference model, $G_{r}(s)$, and a DPOC model, $G_{c}(s)$, that effectively minimised the hook and the payload oscillations, as illustrated in Fig. 2 [34]. The concept of poles-zeros cancellation was used in obtaining $G_{s}(s)$. More specifically, the shaper can be arranged as $G_{s}(s)=G_{r}(s) G_{c}(s)^{-1}$ and formulated as:

$$
G_{s}(s)=\frac{a_{n} s^{n}+a_{n-1} s^{n-1}+a_{n-2} s^{n-2}+\ldots+a_{0}}{\left(s+\omega_{r}\right)^{n}}
$$

where $n$ denotes the system order, $\omega_{r}$ was chosen based upon several tests, and $a_{n}, a_{n-1}$, $a_{n-2}, \ldots, a_{0}$ were the design variables of $G_{s}(s)$. However, the process to obtain the complete 
design variables of an MRCS for a DPOC involved complicated procedures and mathematical formulations, as this was established in previous work [34].

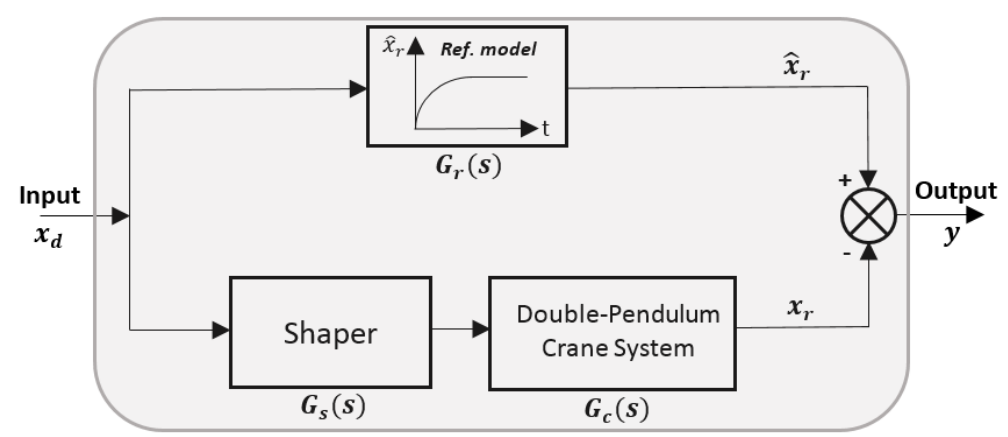

Fig. 2. MRCS block diagram [34]

In an attempt to reduce the design complexity and to ensure that it could be implemented together with a feedback controller, the previously developed MRCS was improved, such that the PSO could be utilised to solve the design variables of the command shaper, $G_{s}(s)$, as illustrated in Fig. 3. By adopting the same concept as the MRCS approach, appropriate values for the numerator, $G_{s}(s)$, as described in Eq. (8), were obtained, so that the poles of the DPOC could be cancelled out, and the overall system poles were only from the denominator of the shaper. Hence, the output value of $x_{r}$ can be expressed as:

$$
x_{r}(t)=\sum_{i=1}^{3} a_{2 i} x_{2 i}(t)
$$

where $a_{2 i}(i=1,2,3)$ are the numerator coefficients, and $x_{2 i}(i=1,2,3)$ are the step responses of $G_{s}(s) G_{c}(s)$.

As the MRCS can only handle hook and payload oscillations, a feedback controller was required, in order to achieve an accurate trolley positioning for the various desired positions. In this work, the PID was designated as a feedback controller, due to its low cost, its easy implementation, and because it is widely used in a number of practical systems [41]. Therefore, a hybrid design of an improved MRCS and PID was required to achieve the optimal 
performance for both of the objectives. As the design variables of $G_{s}(s)$, and the three PID parameters that needed to be obtained concurrently, the benefits of a PSO algorithm were used to simultaneously tune the MRCS-PID controller parameters, as depicted in Fig. 4, which provided an additional advantage for the proposed hybrid scheme.

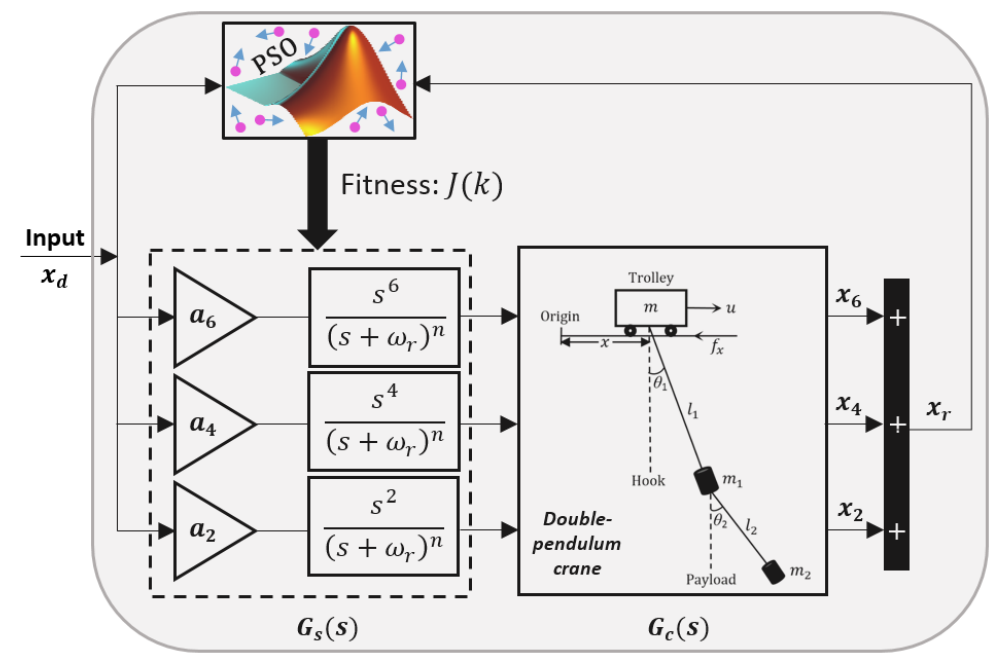

Fig. 3. PSO-based MRCS block diagram

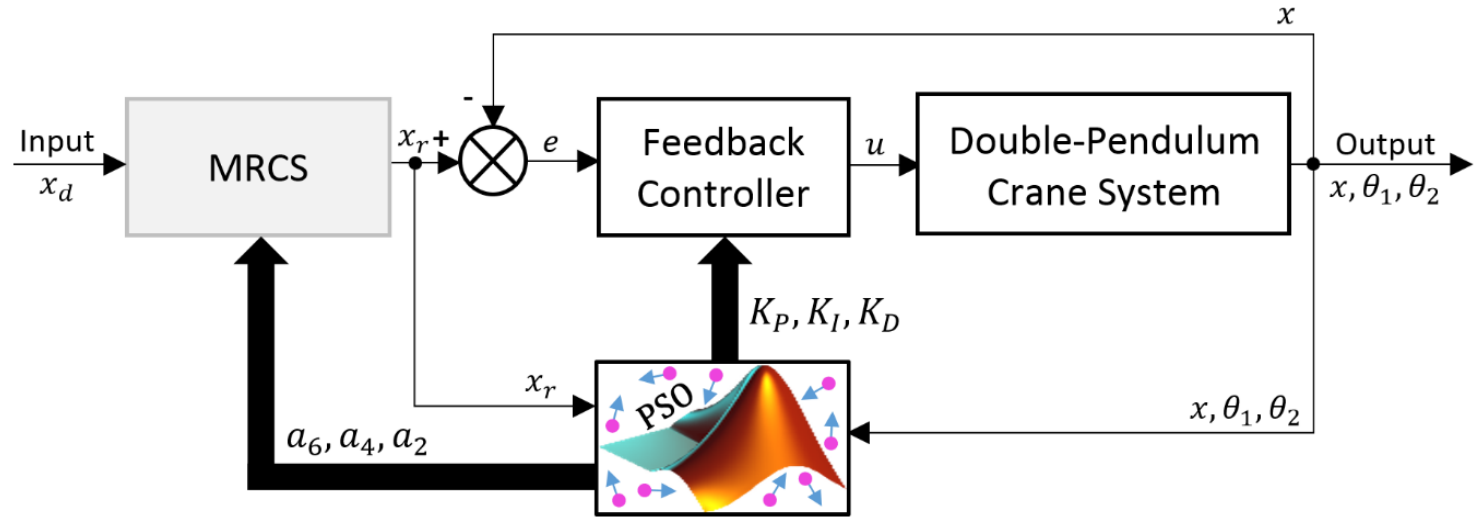

Fig. 4. A hybrid MRCS-PID control block diagram using a PSO algorithm

With a step input of $x_{d}$, and based on the outputs $x, \theta_{1}$, and $\theta_{2}$, the PSO algorithm was formulated to concurrently calculate the six control parameters, which were $a_{6}, a_{4}$, and $a_{2}$ for the MRCS, and $K_{P}, K_{I}$, and $K_{D}$ for the PID controller. When using the MRCS, $x_{d}$ was shaped as $x_{r}$, which was the optimal input reference for the feedback system that theoretically yielded the minimal hook and payload oscillations. For the feedback system, the trolley position of $x$ was the only output used as a feedback signal. In the PSO, two initial parameters of the particle, 
namely, position, $X_{i j}^{k}$ and velocity, $V_{i j}^{k}$ were introduced towards the searching process, where $k, i$, and $j$ were the iteration numbers, the individual swarm, and the dimension of the particle, respectively. The particle $i$ in dimension $j$ for the searching optimisation process can also be defined as $i \in[1, \rho]$ and $j \in[1, D]$, where $\rho$ and $D$ were the entire population and they were high dimensional in a search space. Conceptually, the new particle velocity, $V_{i j}^{k+1}$, was adjusted, according to the $X_{i j}^{k}$, pbest $t_{i j}$, and $g_{b e s t}$ values, and the new particle position, $X_{i j}^{k+1}$, was updated based on $X_{i j}^{k}$ and $V_{i j}^{k+1}$, as in [41-43]:

$$
\begin{gathered}
V_{i j}^{k+1}=w V_{i j}^{k}+c_{1} r_{1}\left(\text { pbest }_{i j}-X_{i j}^{k}\right)+c_{2} r_{2}\left(\text { gbest }_{j}-X_{i j}^{k}\right) \\
X_{i j}^{k+1}=X_{i j}^{k}+V_{i j}^{k+1}
\end{gathered}
$$

where $w$ was chosen for a better exploration and exploitation of the particle that began with 0.9 , and linearly decreased to 0.4 at the maximum iteration, $N$. Furthermore, $c_{1}$ and $c_{2}$ were cognitive and social acceleration coefficients, respectively, while $r_{1}$ and $r_{2}$ represented the random function values, $r_{1}, r_{2} \in U(0,1)$. For the DPOC control, $X_{i j}^{k}$ can also be defined as $\left\{a_{6 i 1}^{k}, a_{4 i 2}^{k}, a_{2 i 3}^{k}, K_{P i 4}^{k}, K_{I i 5}^{k}\right.$, and $\left.K_{D_{i 6}}^{k}\right\}$

To ensure for a precise trolley positioning and higher oscillation reductions for the hook and payload, a fitness function, $J(k)$ that considers the relationship between the trolley position, the hook and payload movements, and the physical parameters of the crane was designed [16]. This concept was originated from the potential energy, $P=m g \Delta h$ and based on vertical distance oscillations of the DPOC system, where a vertical distance of $\Delta h=h_{1}+h_{2}$ as shown in Fig. 5. The $h_{1}$ and $h_{2}$ represent the differences in heights of the hook and payload with respect to a reference height without oscillation, respectively. Lower values of $h_{1}$ and $h_{2}$ indicate low hook and payload oscillations, and directly decrease the potential energy of the system. 


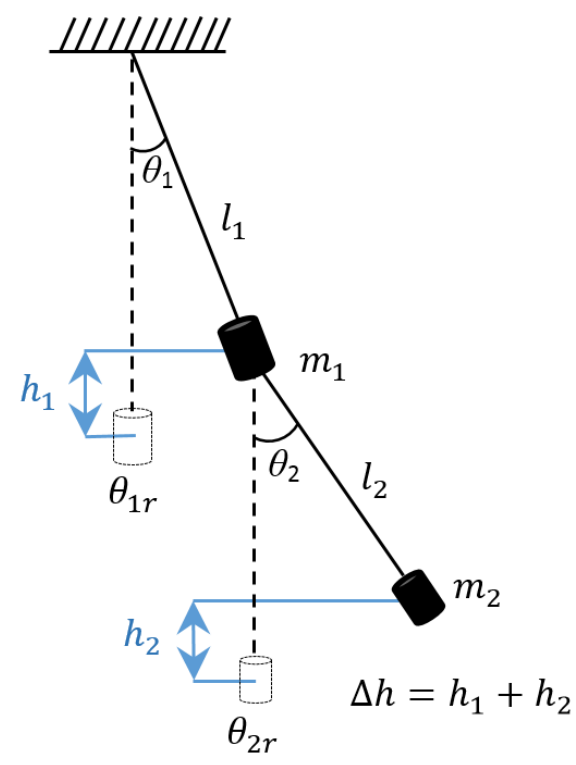

Fig. 5. Vertical distance of hook and payload movements

The performance of each individual particle was assessed by the fitness function and pbest $_{i j}$ and gbest $_{j}$ were updated, if the particle had a minimum $J(k)$ :

$$
\begin{gathered}
J(k)=\sum_{k=1}^{N}\left|x_{r}^{(k)}-x^{(k)}+\Delta h\right| \\
\Delta h=\left(m_{1}+m_{2}\right) g l_{1}\left(\cos \theta_{1 r}-\cos \theta_{1}^{(k)}\right)+m_{2} g l_{2}\left(\cos \theta_{2 r}-\cos \theta_{2}^{(k)}\right)
\end{gathered}
$$

where $\theta_{1 r}=\theta_{2 r}=$ the reference (zero) angles for the hook and the payload. Therefore, the proposed hybrid control parameters varied according to the particle position movements.

\subsection{PID-PID Control Strategy}

In this section, two feedback controllers, namely, PID-PID control were combined and implemented for the DPOC control, and they were used for performance comparison. The technique was recently proposed in [16], and it had the advantage of avoiding the measurement of the payload motion, which is challenging in real practice. The simulation results revealed that the performance was comparable with a full state feedback control, thus, the real-time implementation would be desirable and interesting. 
Fig. 6 shows the block diagram of the PID-PID control scheme. The separate PID feedback controllers were used for position and oscillation control, respectively, by using the feedback signals, $x$ and $\theta_{1}$. Similar to the MRCS-PID control, the PSO algorithm was used to concurrently tune the six PID-PID control parameters, $K_{P 1}, K_{I 1}, K_{D 1}, K_{P 2}, K_{I 2}$, and $K_{D 2}$. A similar fitness function, by replacing $x_{r}$ to $x_{d}$ in Eq. (12), was used without the payload motion sensor. The condition of $\theta_{2}=2 \theta_{1}$, as was designed in [16], was considered for solving the fitness functions in Eqs. (12)-(13). In this case, $X_{i j}^{k}$ was defined as $\left\{K_{P 1_{i 1}}^{k}, K_{I 1_{i 2}}^{k}, K_{D 1_{i 3}}^{k}, K_{P 2 i 4}^{k}\right.$, $K_{I 2}^{k} \underset{i 5}{k}$, and $\left.K_{D 2} \underset{i 6}{k}\right\}$

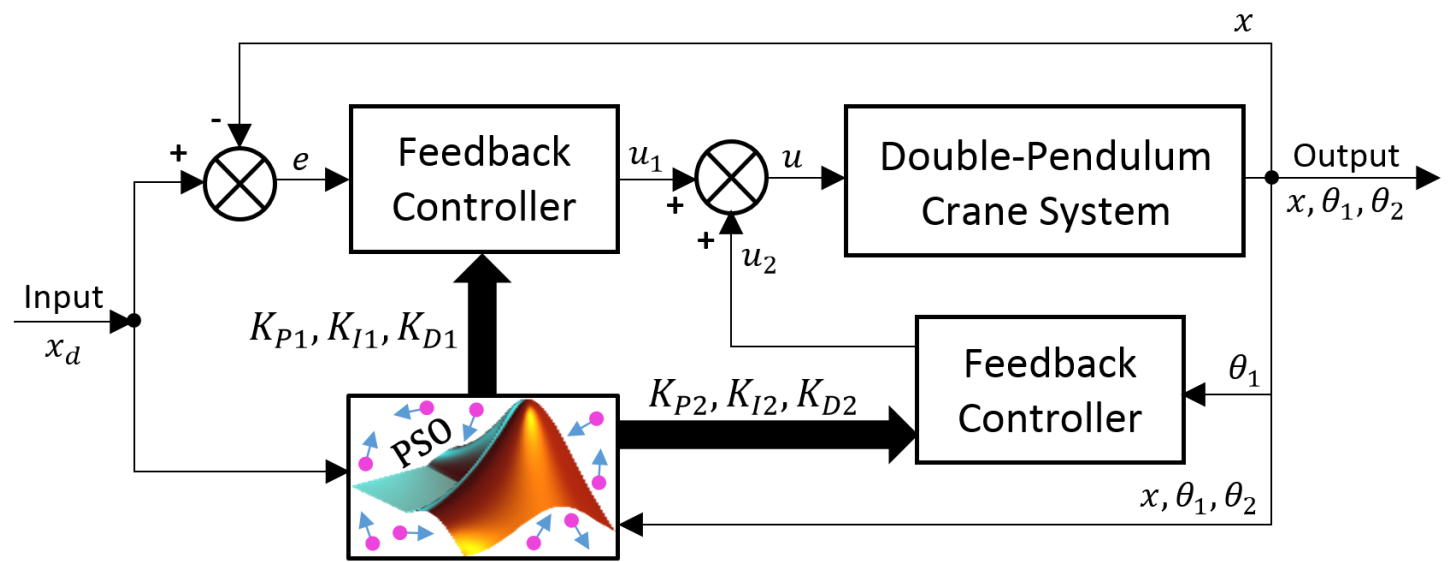

Fig. 6. A PID-PID control block diagram using a PSO algorithm

\section{IMPLEMENTATION AND RESULTS}

In this work, three scenarios that involved different operating conditions of a DPOC were considered: (i) various trolley positions, (ii) payload hoisting, and (iii) payload mass variations. The simulations were performed by using the nonlinear underactuated model of a DPOC, as formulated in Eqs. (1)-(7), whereas the experiments were carried out by using a laboratory DPOC, as shown in Fig. 7, in order to investigate the performance of the proposed MRCS-PID and PID-PID control strategies. The laboratory DPOC was equipped with a trolley, two cables, and two cylindrical loads (dimension: diameter $\times$ height $)$ that represented the hook $(0.06 \mathrm{~m} \times$ $0.076 \mathrm{~m})$ and the payload $(0.05 \mathrm{~m} \times 0.055 \mathrm{~m})$. Three incremental decoders with a resolution of 
4096 pulses per rotation were used for measuring the trolley position, cable length and hook oscillation. In addition, a Logitech C170 camera mounted on the trolley was also used to record the payload motion coordinates based on the deflection of a red indicator located at the top of the payload. The parameters of the laboratory DPOC were: $m=1.155 \mathrm{~kg}, m_{1}=0.20 \mathrm{~kg}, m_{2}=$ $0.10 \mathrm{~kg}, l_{1}=0.30 \mathrm{~m}, l_{2}=0.20 \mathrm{~m}, f_{x}=82 \mathrm{Ns} / \mathrm{m}$, and $g=9.81 \mathrm{~m} / \mathrm{s}^{2}$. These parameters were also used in the simulations.

The correctness of the nonlinear underactuated DPOC model was previously verified in [34], where satisfactory agreements between the simulation and the experimental results were obtained. All of the MRCS-PID and PID-PID control parameters that were designed in Sections 3.1 and 3.2 were used in the simulations and in the real-time implementations, respectively. In order to evaluate the oscillation control performances, maximum amplitudes of the hook, $\theta_{1 m}$, and the payload, $\theta_{2 m}$, angles were measured. In addition, a mean squared error (MSE) was used as a performance index, where low $\mathrm{MSE}_{\theta_{1}}$ and $\mathrm{MSE}_{\theta_{2}}$ values were desirable, as they indicated low overall hook and payload oscillation responses, respectively.

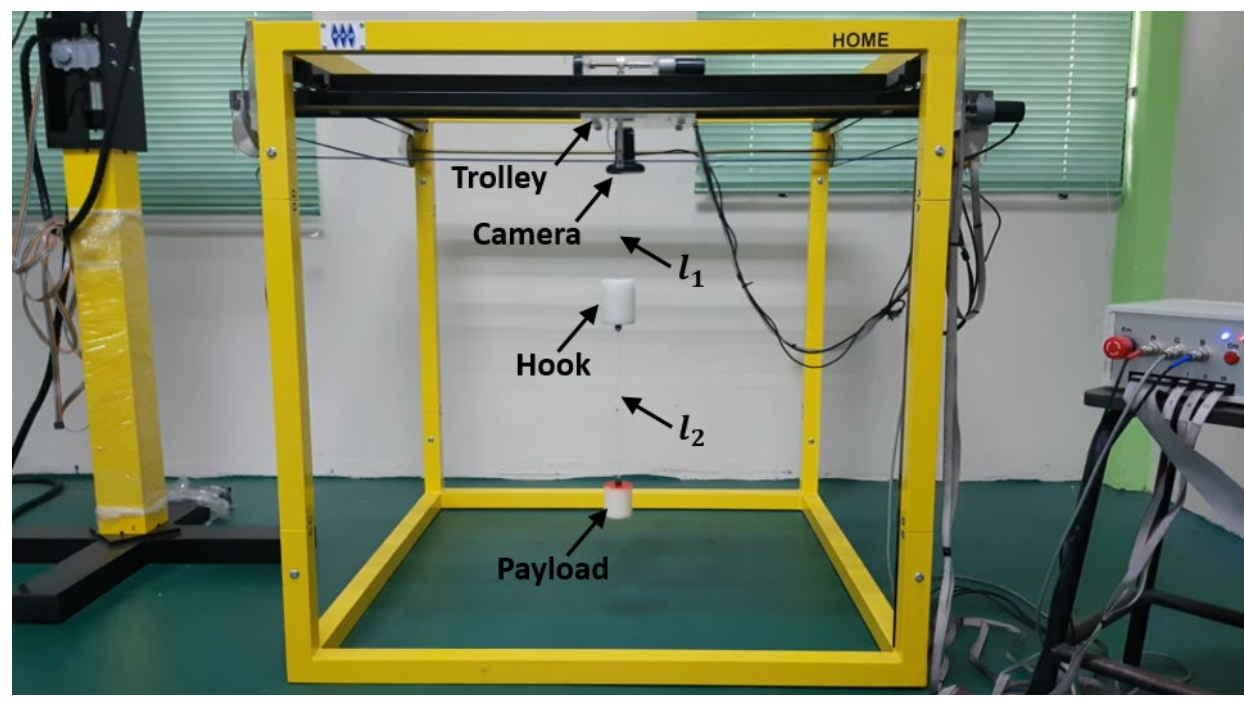

Fig. 7. A laboratory DPOC 


\subsection{Optimal Parameters of the MRCS-PID and PID-PID Control Strategies}

The MRCS-PID and PID-PID control parameters that were based on $x_{d}=0.40 \mathrm{~m}$ were optimally obtained when using the PSO algorithm with a fitness function, as designed in Sections 3.1 and 3.2, respectively, upon the completion of the iteration process. The best practice is to select $\rho$ in between 20 to 50 particles, and with no specific limit of $k$ numbers [44]. On the other hand, in [45-46], the values of $c_{1}$ and $c_{2}$ were suggested as 2 to ensure that the particles attract and influence on the average of pbest $_{i j}$, and gbest $_{j}$ values. Therefore, in this work, $\rho, k, c_{1}$, and $c_{2}$ were set as $20,100,2$, and 2 , respectively. It can be seen that the control parameters varied and remained unchanged, once they reached the 51st and 54th iterations, as shown in Figs. 8 and 9. For the results, the final optimal MRCS-PID control parameters were obtained as $a_{6}=0.1957, a_{4}=43.6252, a_{2}=1063.6992, K_{P}=2.5602, K_{I}=$ 0.0013 , and $K_{D}=0.3555$. Subsequently, the final optimal PID-PID control parameters were obtained as $K_{P 1}=2.2927, K_{I 1}=0.0156, K_{D 1}=1.0401, K_{P 2}=1.1480, K_{I 2}=0.5048$, and $K_{D 2}=$ 0.1314 .

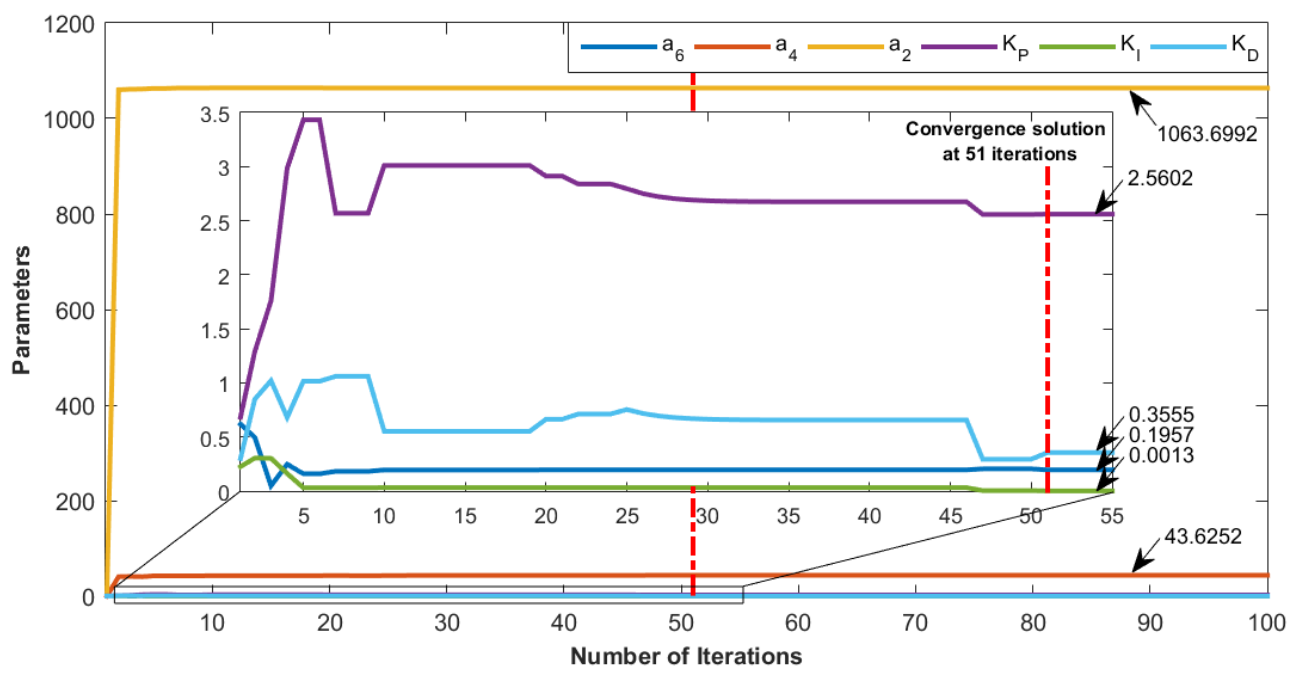

Fig. 8. The optimal MRCS-PID control parameters 


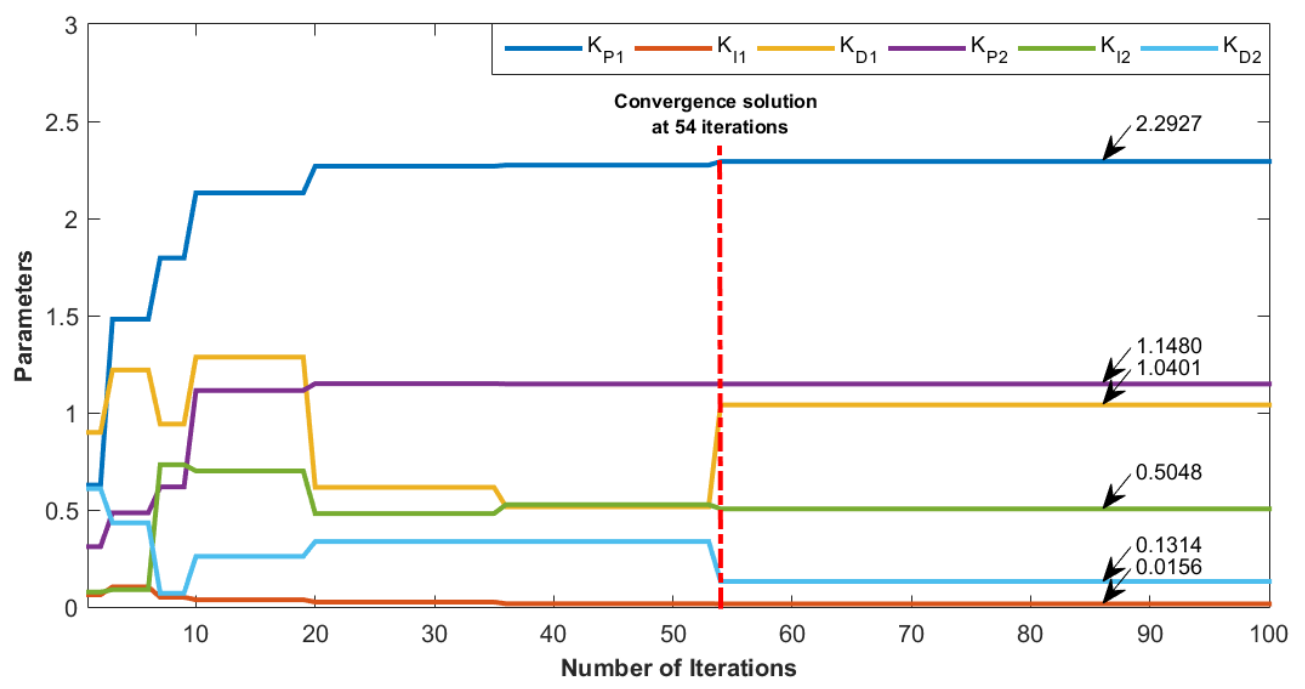

Fig. 9. The optimal PID-PID control parameters

It was important to ensure that all of the poles were in a stable region of the $s$-plane, indicating a closed-loop stable system for the implementations. From Fig. 10, the poles of the proposed MRCS-PID control strategy were located at $p_{1,2}=-3.4 \pm j 0.213, p_{3,4}=$ $-3.04 \pm j 0.482, \quad p_{5,6}=-2.56 \pm j 0.293, \quad p_{7}=-0.0000517, \quad p_{8,9}=-0.118 \pm j 1.31, \quad p_{10,11}=$ $-0.0142 \pm j 5.25$, and $p_{12,13}=-0.0723 \pm j 12.20$. On the other hand, for the PID-PID control strategy, the poles were located at $p_{1}=-0.006826, p_{2,3}=-0.3315 \pm j 1.156, p_{4,5}=$ $-0.1412 \pm j 5.399$, and $p_{6,7}=-0.3775 \pm j 12.26$, as shown in Fig. 11. These demonstrated that the MRCS-PID and PID-PID control parameters that were obtained by using the PSO algorithm provided a stable system, where all of the poles lie on the left half of the $s$-plane. Thus, the optimal control parameters, as shown in Figs. 8 and 9, were ready to be used through the simulation and experimental exercises. 


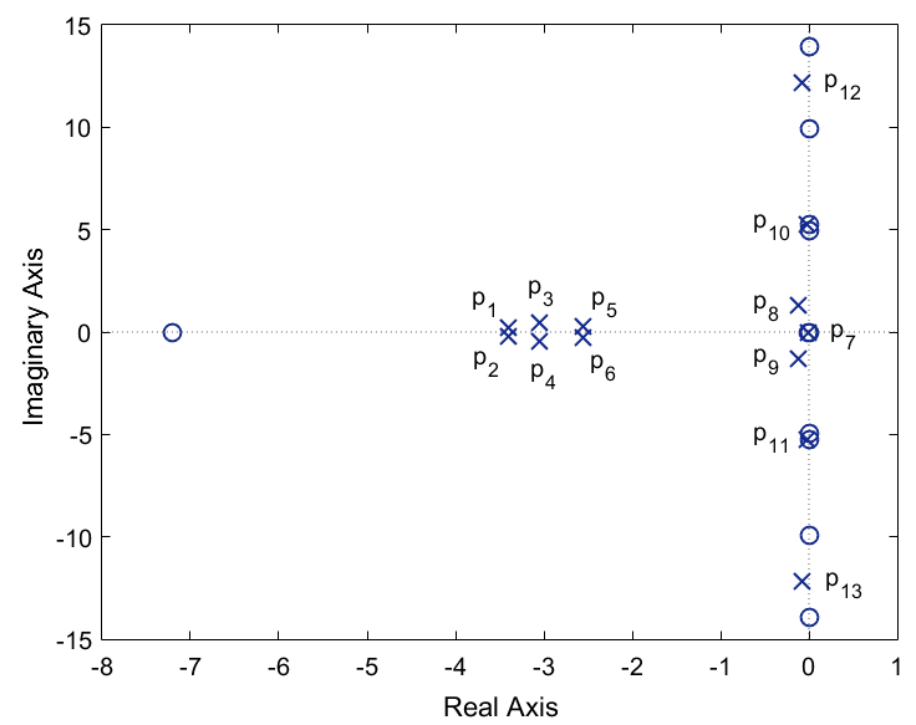

Fig. 10. Pole locations of the MRCS-PID control strategy

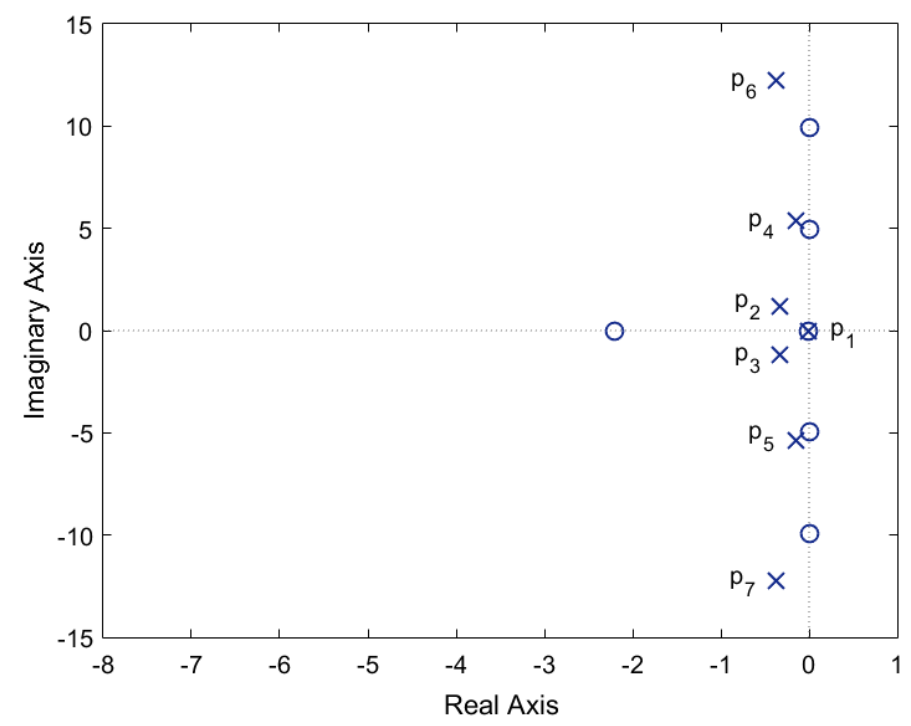

Fig. 11. Pole locations of the PID-PID control strategy

\subsection{Various Trolley Positions}

Initially, the performance of the proposed MRCS-PID control was investigated by the unshaped control that was generated only by using the PID controller (bypassing the MRCS in Fig. 4), in order to ensure that the trolley reached the desired position. This methodology was also presented in [39], so as to observe the effectiveness of an MRCS as an oscillations control. In Fig. 12, with the desired trolley position, $x_{d}=0.40 \mathrm{~m}$, both of the simulations (Sim) and the experiments (Exp) yielded a similar pattern of trolley position responses for the unshaped and 
MRCS-PID controllers. In the experiments, the trolley reached the destination within $4.57 \mathrm{~s}$ and $6.62 \mathrm{~s}$, by using the unshaped and MRCS-PID controllers, respectively. It was noted that the unshaped control provided a $31 \%$ faster response than the proposed strategy.

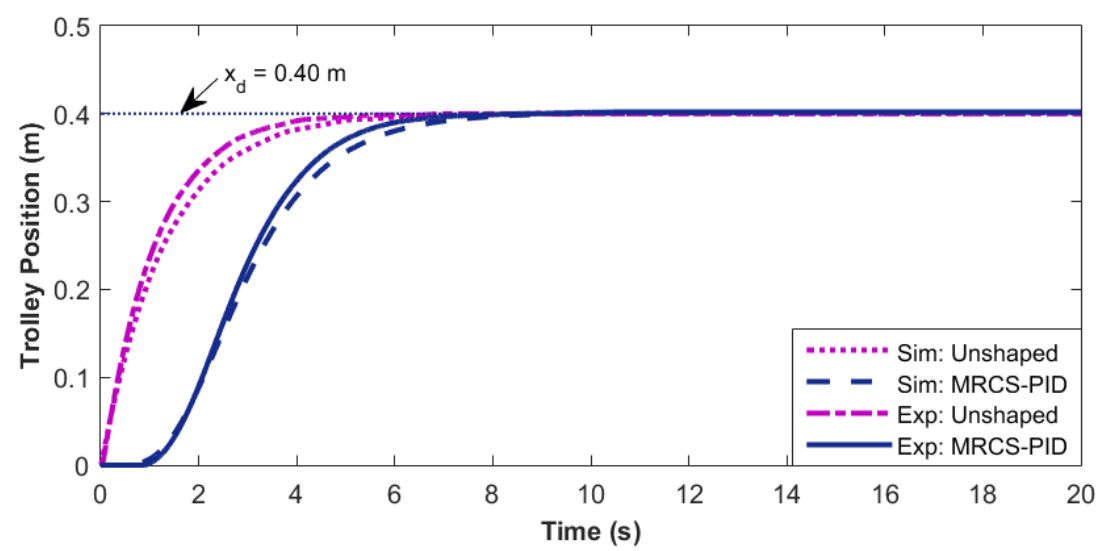

Fig. 12. Trolley movement when $x_{d}=0.40 \mathrm{~m}$

Unfortunately, the fast motion response of the unshaped control suffered from higher hook and payload oscillation responses, as presented in Figs. 13 and 14. Meanwhile, the hook and the payload continuously oscillated with large oscillations, even after the trolley reached the desired location, which then brought forth a safety concern. In the experiments, the unshaped control provided $\theta_{1 m}, \theta_{2 m}, \mathrm{MSE}_{\theta_{1}}$, and $\mathrm{MSE}_{\theta_{2}}$ being as large as $10.635^{\circ}, 12.810^{\circ}, 20.984$, and 46.855 , respectively, whereas the proposed method provided $1.046^{\circ}, 2.430^{\circ}, 0.146$, and 0.328 , as shown in Fig. 14. It was found that the MRCS-PID control provided a significant oscillation reduction of $86.8 \%$ and $81 \%$ in $\theta_{1 m}$ and $\theta_{2 m}$, respectively, when compared to the unshaped control. Furthermore, the hook and the payload oscillations were efficiently attenuated and eliminated within $3 \mathrm{~s}$. This has indicated that the implementation of an MRCS into a hybrid control strategy contributes to a significant impact in minimising both of the oscillations. 


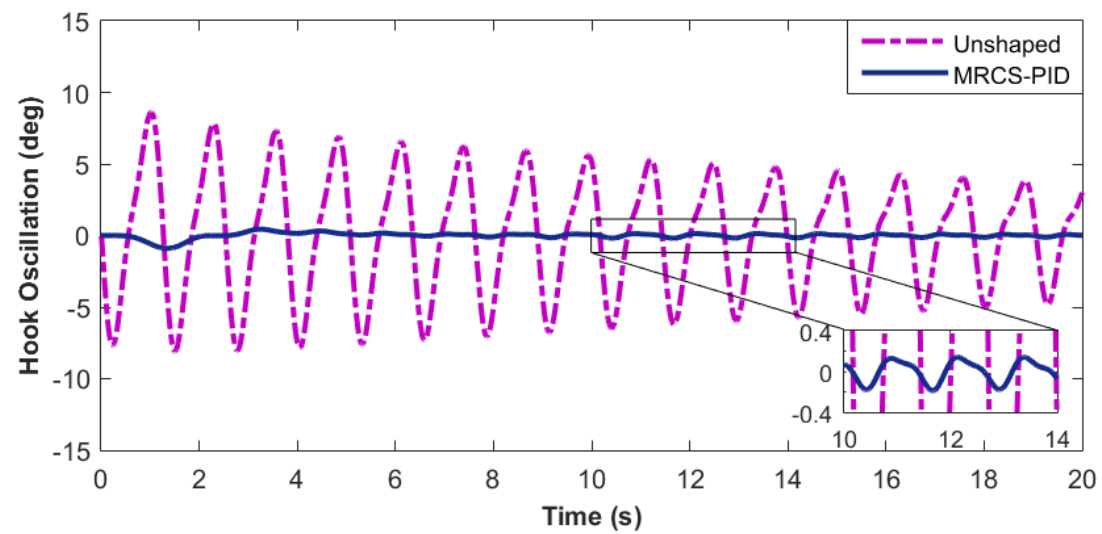

(a)

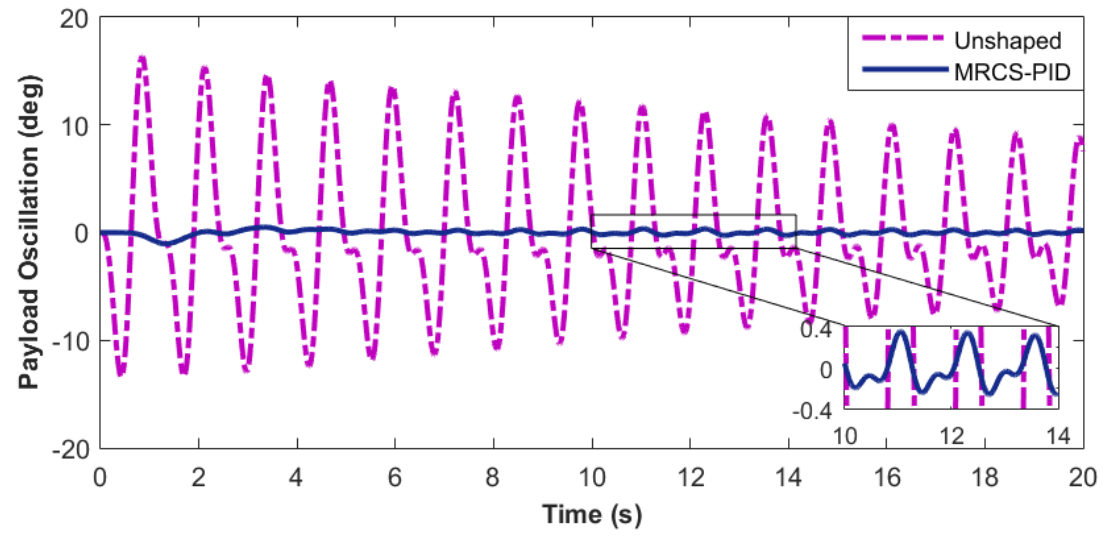

(b)

Fig. 13. Simulation responses of a DPOC when $x_{d}=0.40$ m: (a) Hook (b) Payload

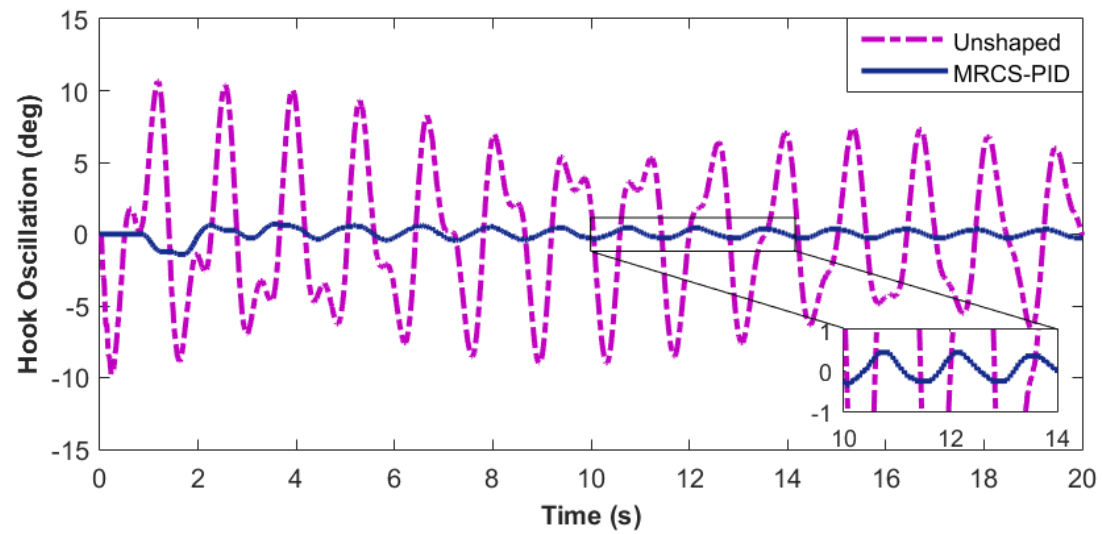

(a) 


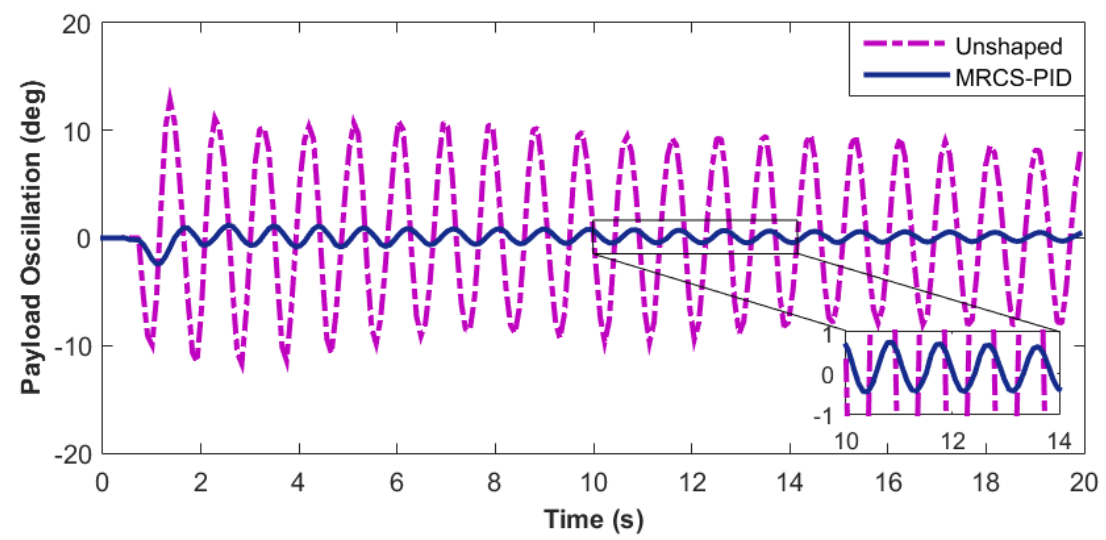

(b)

Fig. 14. Experimental responses of a DPOC when $x_{d}=0.40 \mathrm{~m}$ : (a) Hook (b) Payload

The effectiveness of the MRCS-PID tracking control against various $x_{d}$ was next investigated. The trolley was required to move $\pm 0.20 \mathrm{~m}$ from the position in the previous investigation, which were the lower $\left(x_{d}=0.20 \mathrm{~m}\right)$ and higher $\left(x_{d}=0.60 \mathrm{~m}\right)$ positions. The proposed MRCS-PID successfully positioned the trolley accurately, even though the controller parameters were kept constant, as plotted in Fig. 15. The results also showed a similar pattern of trolley positions throughout the simulations and the experiments. In the experiments, the trolley successfully reached the lower and higher positions within $5.81 \mathrm{~s}$ and $8.13 \mathrm{~s}$, respectively.

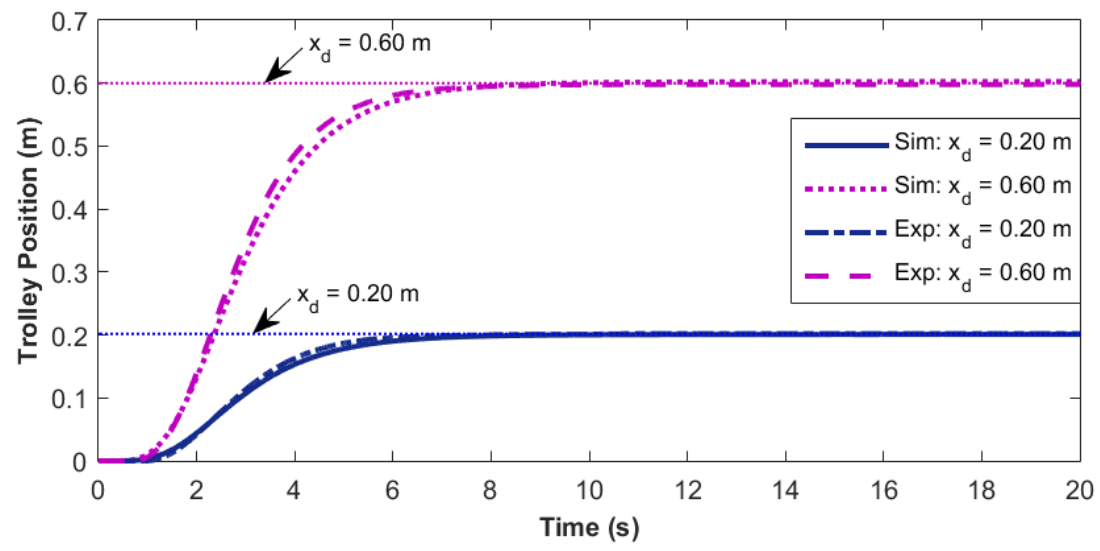

Fig. 15. Trolley position responses with various $x_{d}$ 
As a higher desired position results in a higher control input, the hook and the payload oscillations when $x_{d}=0.60 \mathrm{~m}$ were higher than in the case when $x_{d}=0.20 \mathrm{~m}$. This can be seen in Figs. 16 and 17. The overall performances of the hook and the payload using different trolley positions are summarised in Fig. 18. The effectiveness and the robustness of the MRCS-PID control were further investigated, by comparing with the PID-PID control under scenarios (ii) and (iii), in Sections 4.3 and 4.4, respectively.

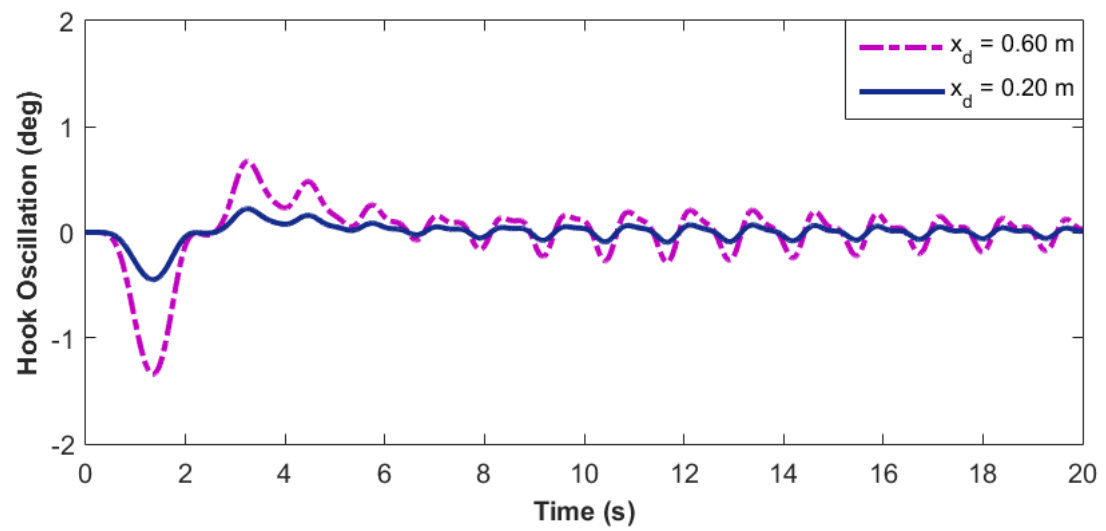

(a)

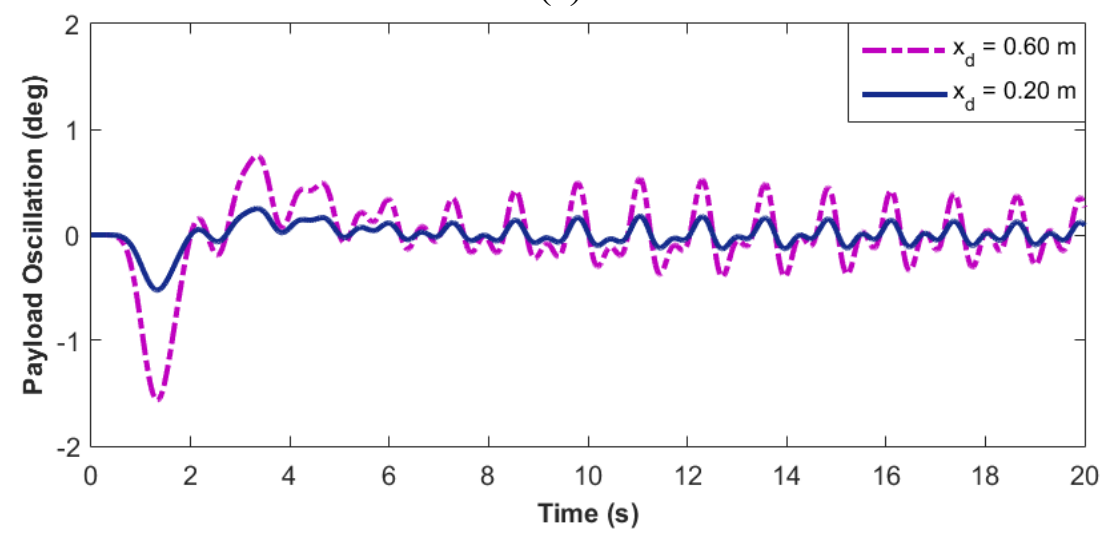

(b)

Fig. 16. Simulation responses of a DPOC at various $x_{d}$ : (a) Hook (b) Payload 


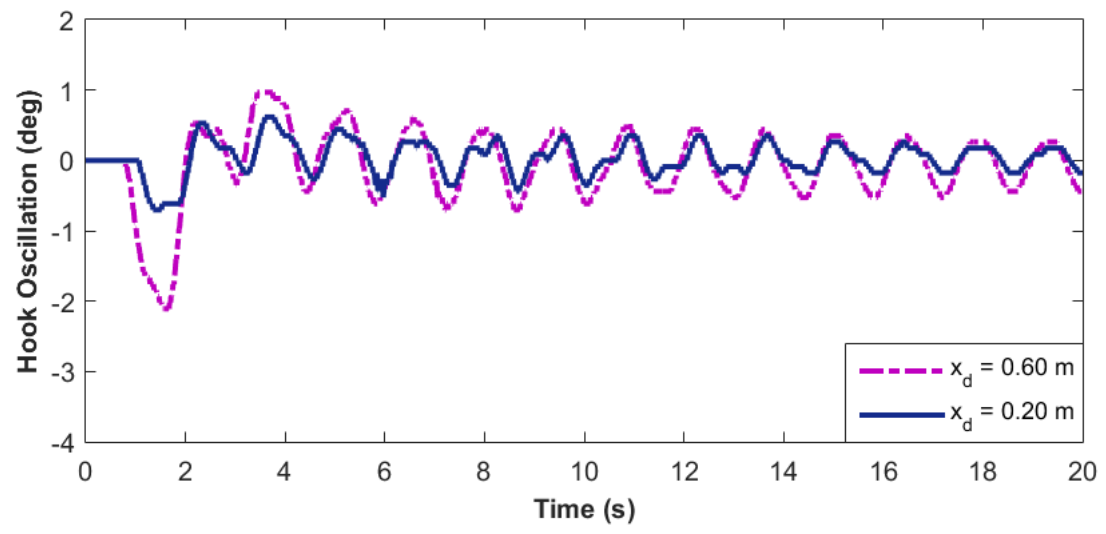

(a)

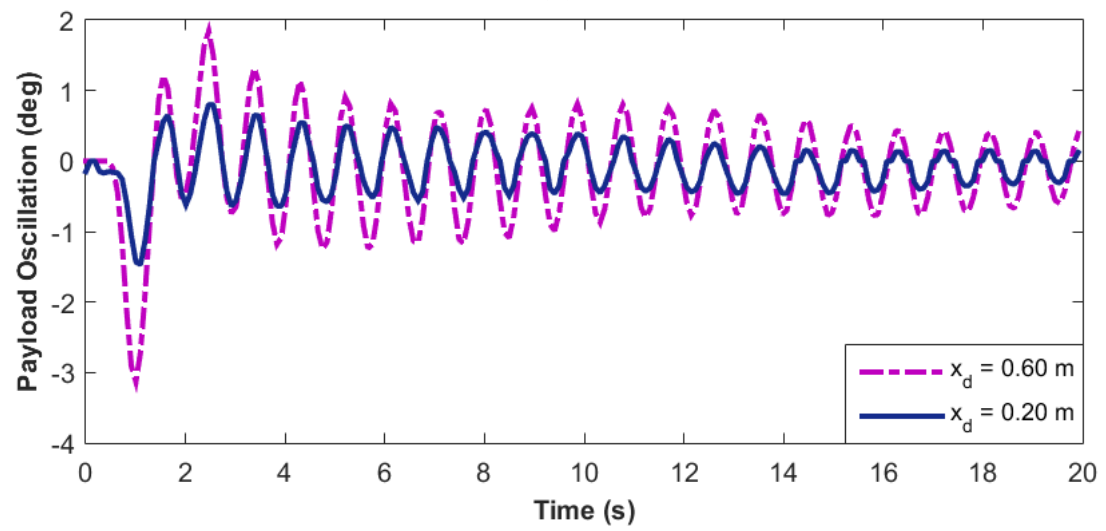

(b)

Fig. 17. Experimental responses of a DPOC at various $x_{d}$ : (a) Hook (b) Payload

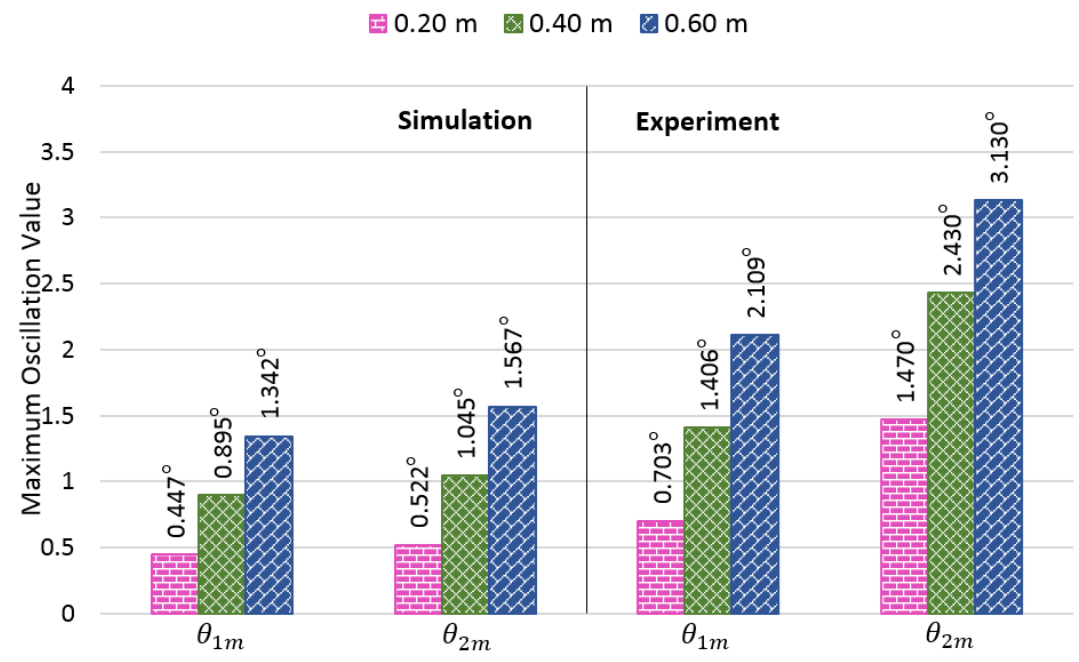

(a) 


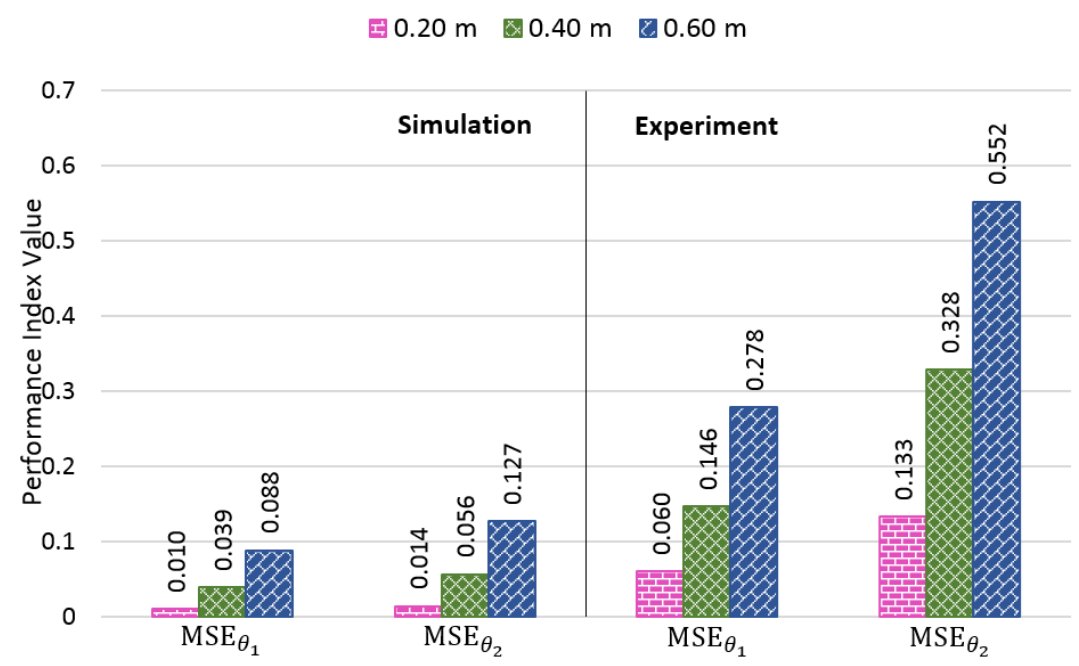

(b)

Fig. 18. Performance of the controller at various $x_{d}$ : (a) Maximum oscillation (b) Overall oscillation

\subsection{Payload Hoisting}

In this work, the effectiveness of the proposed hybrid control was evaluated for a case of varying cable lengths during the payload hoisting, as this operation is essential in industries. The same DPOC parameters as in Section 4 were used, but $l_{1}$ was continuously hoisted from $0.20 \mathrm{~m}$ to $0.40 \mathrm{~m}\left(l_{1}=0.20-0.40 \mathrm{~m}\right)$ and $f_{l}=75 \mathrm{Ns} / \mathrm{m}$. It was clearly noted that the payload reached $0.40 \mathrm{~m}$ in $2.09 \mathrm{~s}$ and $2.24 \mathrm{~s}$ in the simulation and in the experiment, respectively, as depicted in Fig. 19.

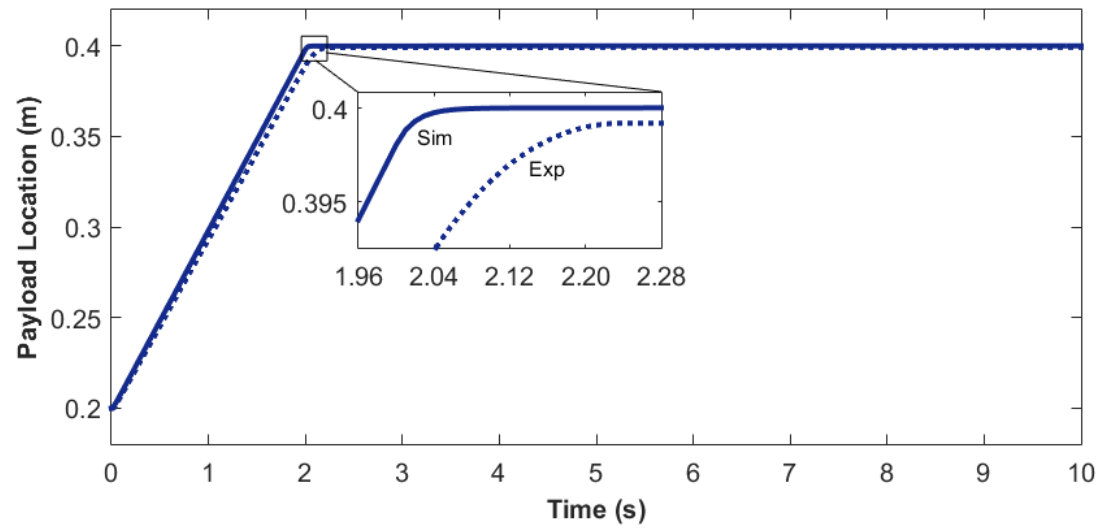

Fig. 19. Varying cable lengths during the payload hoisting $\left(l_{1}=0.20-0.40 \mathrm{~m}\right)$ 
In these cases, the MRCS-PID control was further investigated by comparing with a PIDPID feedback control, as shown in Fig. 6. The parameters of the MRCS-PID control remained unchanged, as in Fig. 8, while the PID-PID control parameters that were obtained in Fig. 9 were used for both the simulation and the experiment implementations. Since the higher desired position induced the higher oscillation responses, as demonstrated in Figs. 16-18, $x_{d}=0.60 \mathrm{~m}$ was selected as an extreme condition, in order to investigate the effectiveness and the robustness of the proposed method under payload hoisting. The MRCS-PID and PID-PID control strategies yielded the control inputs, $u$, as shown in Fig. 20.

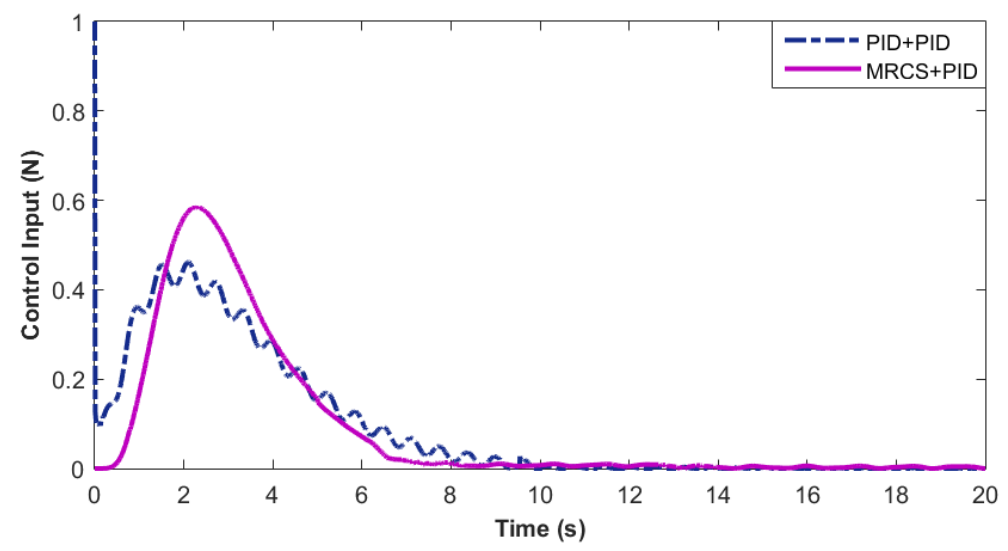

Fig. 20. Control inputs for the MRCS-PID and PID-PID control strategies

As expected, both of the MRCS-PID and PID-PID control strategies that were using optimal control parameters, successfully moved the trolley to the desired location accurately, as shown in Fig. 21. For the MRCS-PID control when using a smoother control input, as shown in Fig. 20, the experimental results showed that the trolley was able to reach the desired position within 8.39 s. Noticeably, this response was $15.9 \%$ faster than the PID-PID control that required $9.98 \mathrm{~s}$ with a visible jerk motion, as zoomed-in Fig. 21. In addition, the smoother control input in the MRCS-PID control strategy resulted in a less actuator effort. 


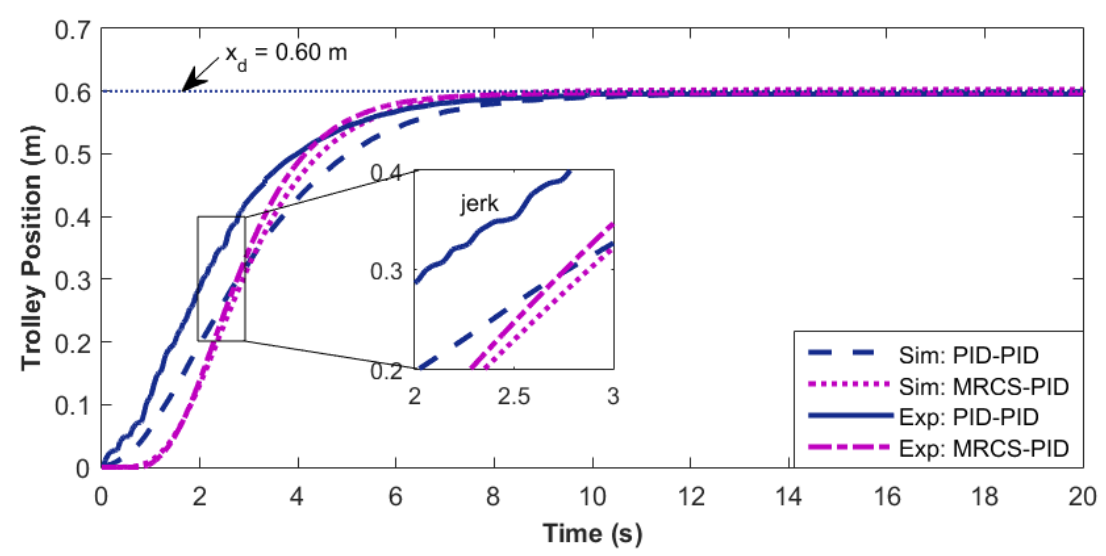

Fig. 21. Trolley position at $l_{1}=0.20-0.40 \mathrm{~m}$

Moreover, both of the oscillation results in the simulation and in the experimental were also recorded in Figs. 22 and 23, respectively. The jerk motion in the PID-PID control disrupted the hook oscillation over the first four seconds, as exhibited in Fig. 23(a). In contrast to the case in Section 4.2, the payload motion was measured experimentally in centimetres. In fact, the PID-PID control resulted in higher $\theta_{1 m}, \theta_{2 m}, \mathrm{MSE}_{\theta_{1}}$, and $\mathrm{MSE}_{\theta_{2}}$ that reached $4.922^{\circ}, 5.030$ $\mathrm{cm}, 2.830$, and 5.192, respectively, as recorded in Table 1. With further observations, the MRCS-PID achieved $2.022^{\circ}, 3.920 \mathrm{~cm}, 0.217$, and 0.488 , with reductions of $58.9 \%, 22.1 \%$, $92.3 \%$, and $90.6 \%$ in $\theta_{1 m}, \theta_{2 m}, \mathrm{MSE}_{\theta_{1}}$, and $\mathrm{MSE}_{\theta_{2}}$, respectively, when compared to the results of the PID-PID control.

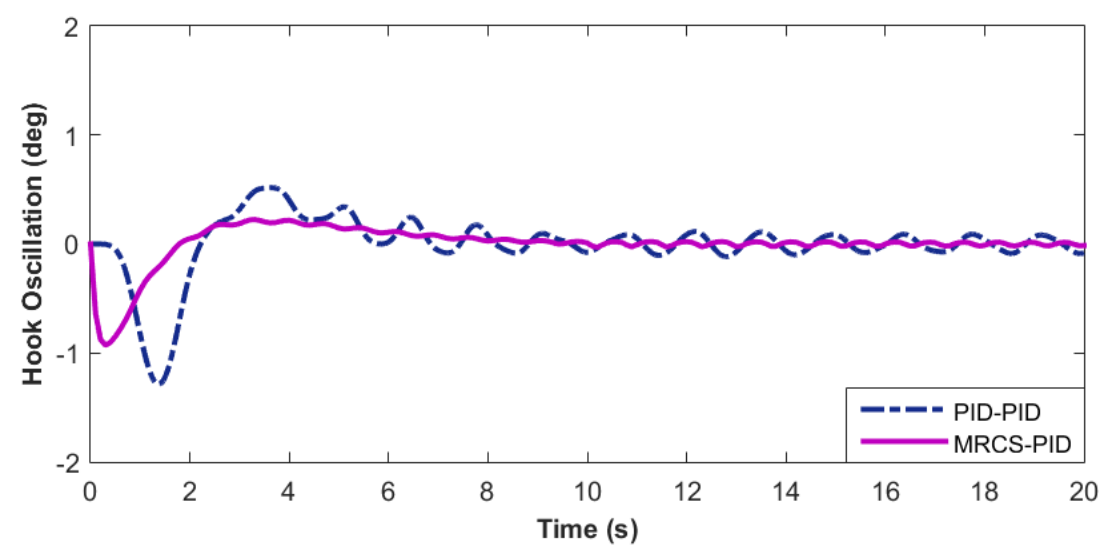

(a) 


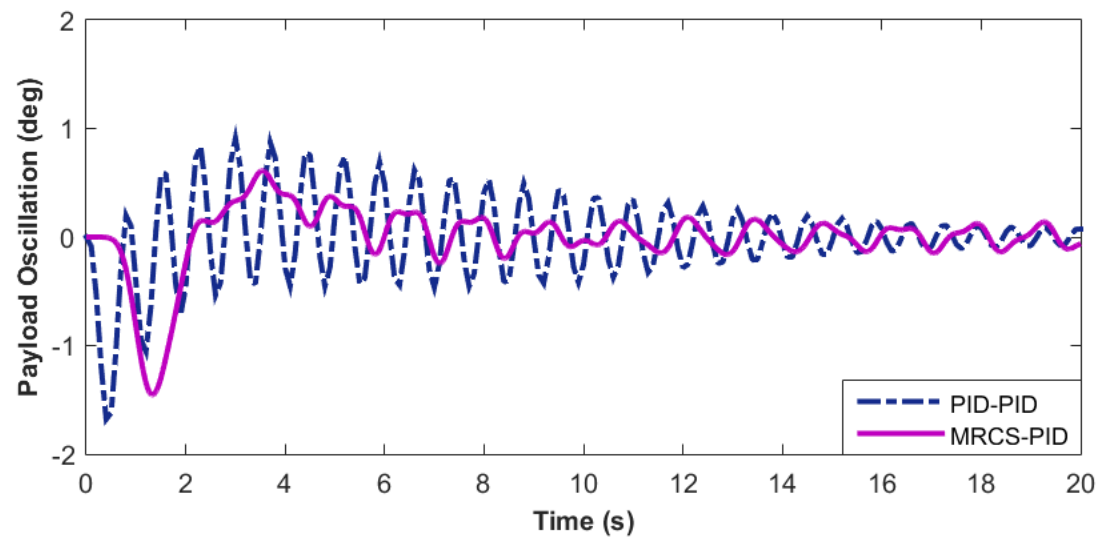

(b)

Fig. 22. Simulation responses of a DPOC when $l_{1}=0.20-0.40 \mathrm{~m}$ : (a) Hook (b) Payload

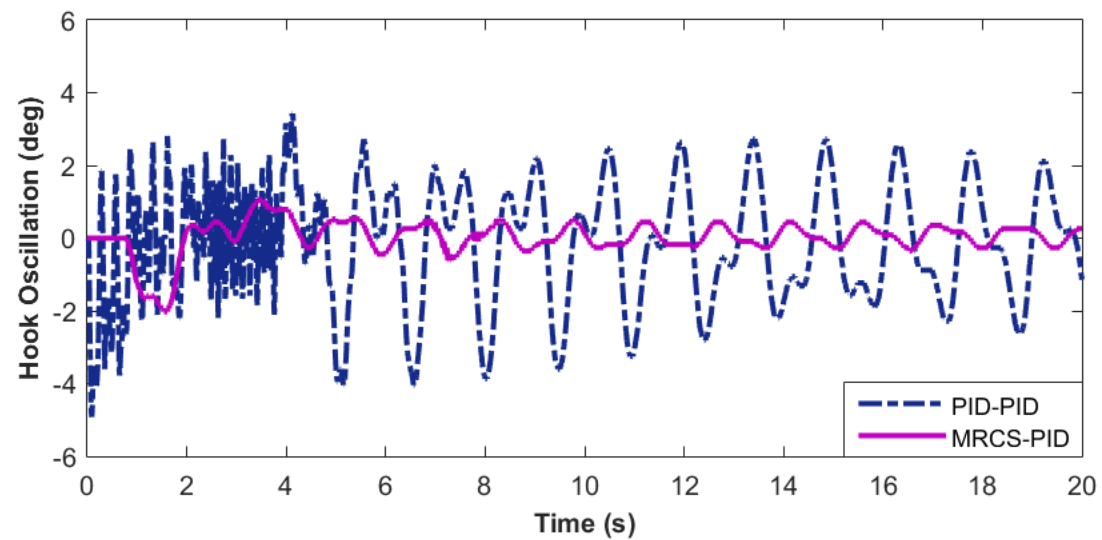

(a)

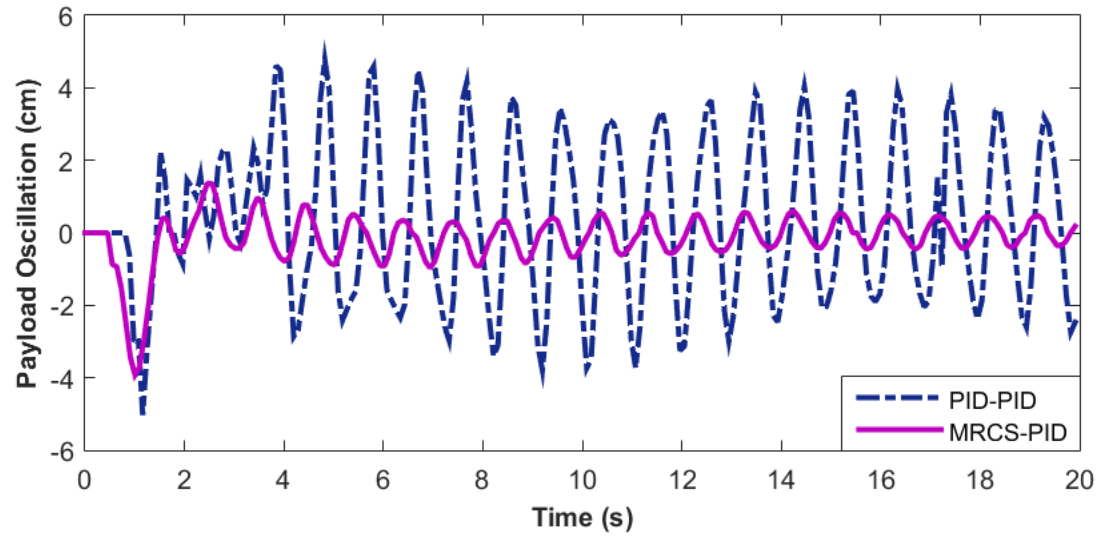

(b)

Fig. 23. Experimental responses of a DPOC when $l_{1}=0.20-0.40 \mathrm{~m}$ : (a) Hook (b) Payload 
Table 1. Performance of the control strategies when $l_{1}=0.20-0.40 \mathrm{~m}$

\begin{tabular}{cccccc}
\hline & Method & $\theta_{1 m}$ & $\theta_{2 m}$ & MSE $_{\theta_{1}}$ & MSE $_{\theta_{2}}$ \\
\hline \multirow{3}{*}{$\operatorname{Sim}$} & PID-PID & $1.286^{\circ}$ & $1.678^{\circ}$ & 0.078 & 0.149 \\
& MRCS-PID & $0.929^{\circ}$ & $1.454^{\circ}$ & 0.035 & 0.092 \\
\cline { 2 - 6 } & $\%$ Reduction & $27.8 \%$ & $13.3 \%$ & $55.1 \%$ & $38.3 \%$ \\
\hline \multirow{3}{*}{ Exp } & PID-PID & $4.922^{\circ}$ & $5.030 \mathrm{~cm}$ & 2.830 & 5.192 \\
& MRCS-PID & $2.022^{\circ}$ & $3.920 \mathrm{~cm}$ & 0.217 & 0.488 \\
\cline { 2 - 6 } & $\%$ Reduction & $58.9 \%$ & $22.1 \%$ & $92.3 \%$ & $90.6 \%$ \\
\hline
\end{tabular}

\subsection{Payload Hoisting with Payload Mass Variations}

From a practical perspective, the payload changed under the circumstances of payload mass variations. Using $x_{d}=0.60 \mathrm{~m}$, the robustness of the MRCS-PID and the PID-PID control schemes was further investigated, by considering a payload, $m_{2}=0.20 \mathrm{~kg}$, which was twice the amount of the previous investigation. From Fig. 24, even using a different payload mass, the proposed method successfully positioned the trolley at the desired position. Similarly, the simulation and the experimental responses showed that the PID-PID control resulted in much larger hook and payload oscillations, as shown in Figs. 25 and 26, respectively. The overall effects of the payload, $m_{2}=0.20 \mathrm{~kg}$ are recorded in Table 2 . Specifically, the experimental results revealed that the MRCS-PID control was found to be superior at $\theta_{1 m}, \theta_{2 m}, \mathrm{MSE}_{\theta_{1}}$, and $\mathrm{MSE}_{\theta_{2}}$, with reductions of $52.7 \%, 36.6 \%, 86.4 \%$, and $83.8 \%$, respectively, under payload hoisting with a higher payload mass. 


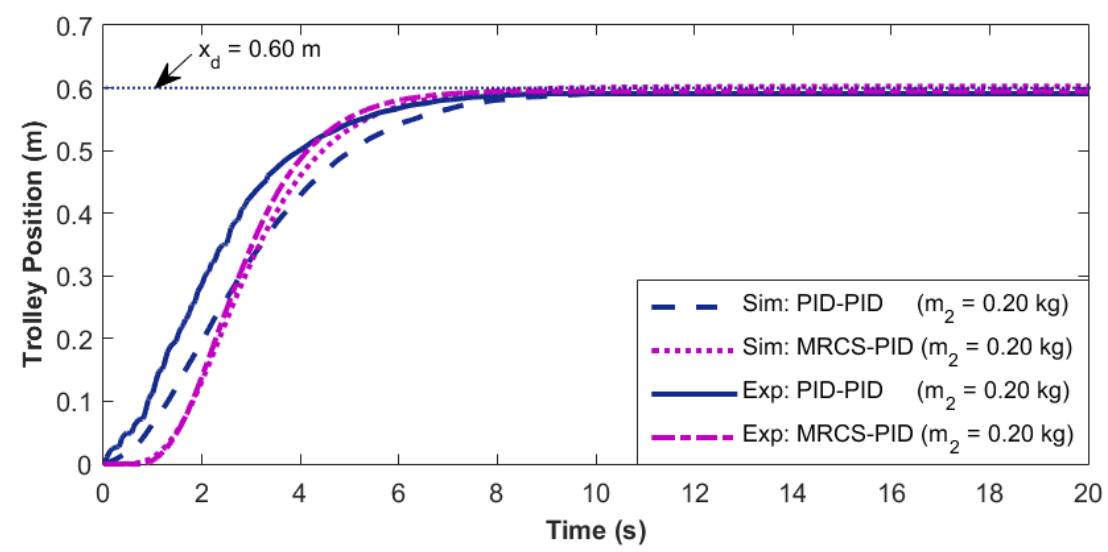

Fig. 24. Trolley position, with payload hoisting and a different payload mass

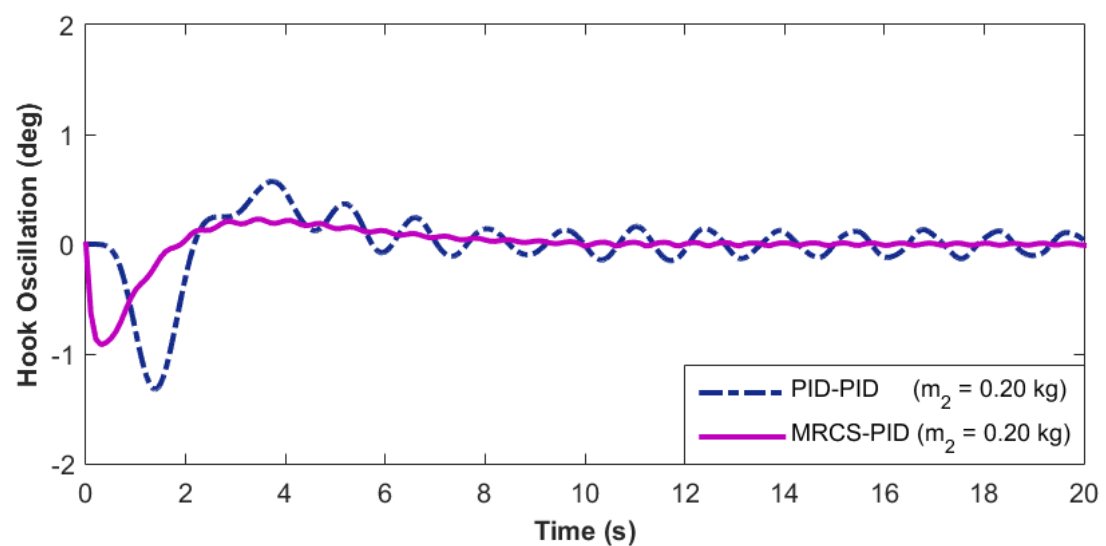

(a)

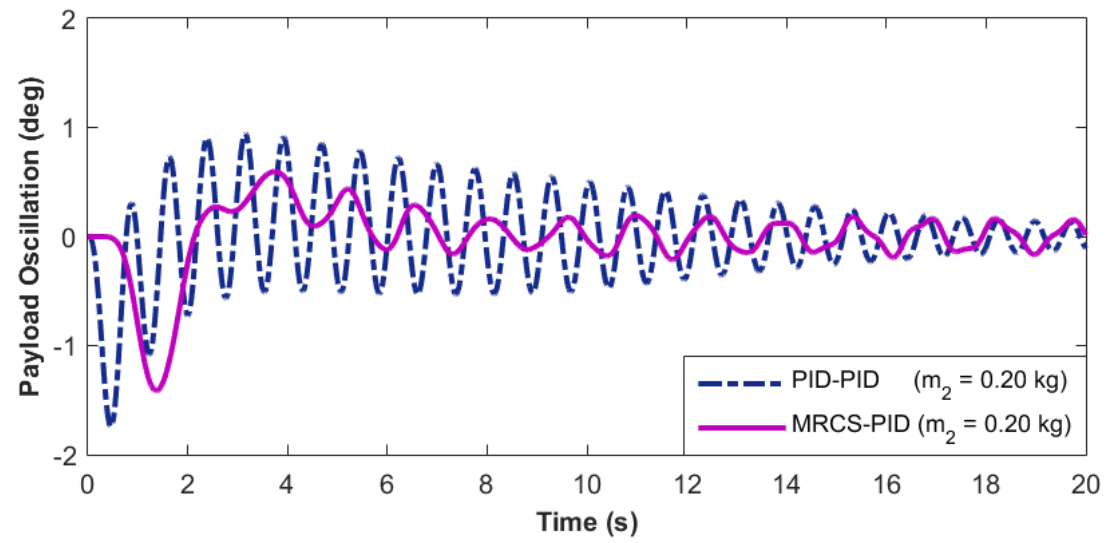

(b)

Fig. 25. Simulation responses of a DPOC when $m_{2}=0.20 \mathrm{~kg}$ : (a) Hook (b) Payload 


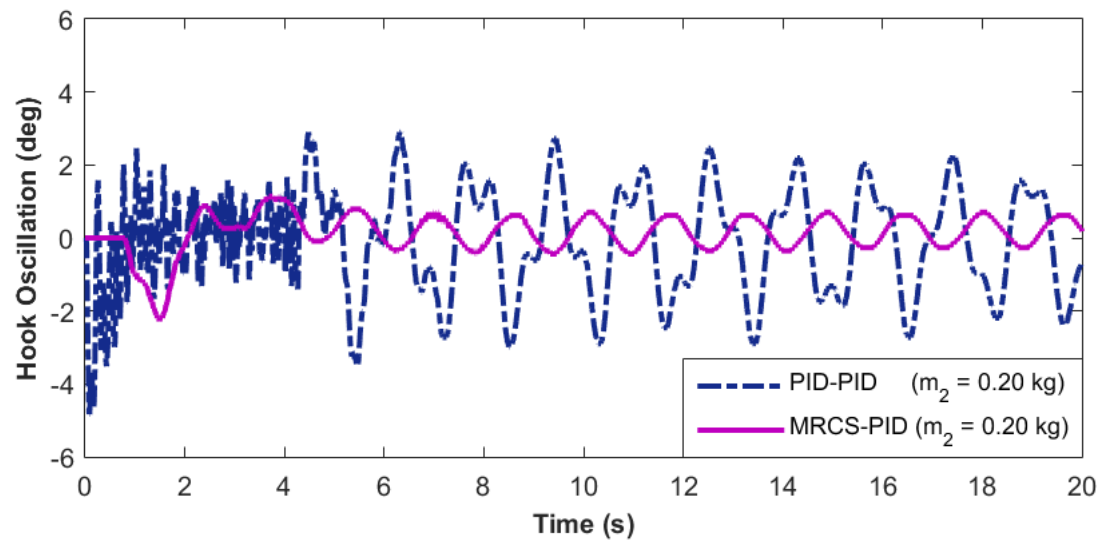

(a)

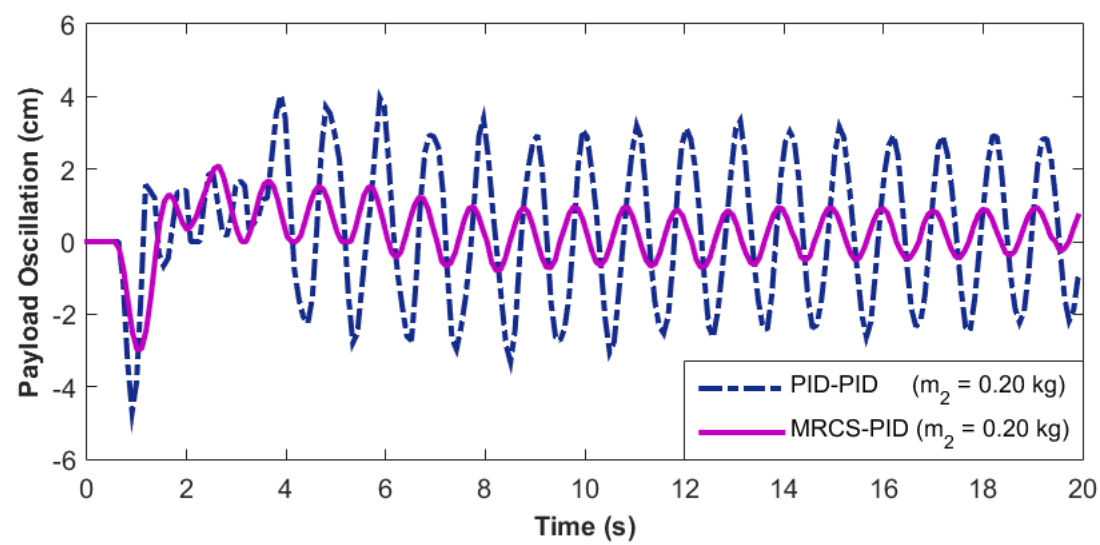

(b)

Fig. 26. Experimental responses of a DPOC when $m_{2}=0.20 \mathrm{~kg}$ : (a) Hook (b) Payload

Table 2. Performance of the controllers when $m_{2}=0.20 \mathrm{~kg}$

\begin{tabular}{cccccc}
\hline & Method & $\theta_{1 m}$ & $\theta_{2 m}$ & MSE $_{\theta_{1}}$ & MSE $_{\theta_{2}}$ \\
\hline \multirow{3}{*}{$\operatorname{Sim}$} & PID-PID & $1.321^{\circ}$ & $1.745^{\circ}$ & 0.085 & 0.176 \\
& MRCS-PID & $0.916^{\circ}$ & $1.415^{\circ}$ & 0.035 & 0.095 \\
\cline { 2 - 6 } & $\%$ Reduction & $30.7 \%$ & $18.9 \%$ & $58.8 \%$ & $46 \%$ \\
\hline \multirow{3}{*}{ Exp } & PID-PID & $4.834^{\circ}$ & $4.700 \mathrm{~cm}$ & 2.190 & 4.034 \\
\cline { 2 - 6 } & MRCS-PID & $2.285^{\circ}$ & $2.980 \mathrm{~cm}$ & 0.297 & 0.653 \\
\cline { 2 - 6 } & $\%$ Reduction & $52.7 \%$ & $36.6 \%$ & $86.4 \%$ & $83.8 \%$ \\
\hline
\end{tabular}

\subsection{Other Control Issues}

For all scenarios, it was noted that the hook and payload oscillations obtained using the MRCSPID control in experiments (Figs. 14, 17, 23 and 36) were slightly larger than the simulation results (Figs. 13, 16, 22 and 25). This might be due to the friction which was ignored in the 
simulation. Nevertheless, the experimental hook and payload oscillations were considerably small, less than 1 degree.

By using the optimal parameters of the command shaper and the feedback controller obtained with the PSO, the MRCS-PID control was shown to be robust to the crane parameter uncertainties involving varying cable lengths and payload mass variations. Lower and satisfactory hook and payload oscillations were achieved under these cases as compared to other approaches. However, the proposed control structure especially relating to the MRCS is not robust to the influence of external disturbances such as wind. To handle the disturbances, a scheme that can automatically update the hybrid controller has to be developed. One of the techniques which can be explored in the future is as presented in $[5,46]$, in which a neural network was used to predict and update the input shaping parameters online.

In this work, the cable of DPOC system is considered to be rigid, with the assumption that it does not bend during payload swing. As described in [47], in certain cases, cable flexibility occurs that will exhibit flexible characteristics and introduce bending deformation during payload oscillation. This may result in a larger amplitude payload oscillation, similar to the effect of external disturbance. In addition, the natural frequency of the DPOC may also be affected. As the proposed MRCS-PID is designed based on optimal constant command shaper parameters and feedback controller gains, the system performance will be affected. In future, it will be interesting to explore the changes in the system dynamics of the DPOC due to the cable flexibility, and to design an improved MRCS controller to handle this issue.

\section{CONCLUSION}

A new control structure using an improved MRCS, together with a practical feedback controller (MRCS-PID), was designed for accurate trolley positioning and oscillation control for an underactuated DPOC under parameter uncertainties. The existing MRCS algorithm was improved, in order to reduce the design complexity and to ensure concurrent tuning with the 
feedback controller can be performed. This was realised using the PSO algorithm, which has not been implemented in the existing literature. In addition, the advantage of an MRCS, which does not require prior knowledge of crane oscillation frequencies, was retained. The proposed approach was demonstrated to be robust under the parameter uncertainties, with respect to the changes in crane dynamics that resulted from the various desired trolley positions, the payload hoisting, and the payload mass variations. Under these conditions, higher reductions in the maximum and overall oscillations were attained when compared to the PID-PID control, in both of the simulations and the experiments. It is envisaged that the improved MRCS can be further utilised for control of other underactuated systems with a higher system order. These include a multi-link flexible robot manipulator, various types of double-pendulum crane and a drone with a cable-suspension payload. The vibrations and oscillations occur in these systems involve with several modes of frequencies and damping ratios.

\section{ACKNOWLEDGEMENT}

The authors would like to sincerely thank the Universiti Teknologi Malaysia, Universiti Teknikal Malaysia Melaka and Ministry of Higher Education, Malaysia for the financial support through Fundamental Grant Research Scheme FRGS/1/2018/TK04/UTM/02/38 and Vote No. 5F044.

\section{REFERENCES}

[1] L.A. Tuan, Fractional-order fast terminal back-stepping sliding mode control of crawler cranes, Mech. Mach. Theory. 137 (2019) 297-314.

[2] L. Ramli, Z. Mohamed, A.M. Abdullahi, H.I. Jaafar, I.M. Lazim, Control strategies for crane systems: A comprehensive review, Mech. Syst. Signal Process. 95 (2017) 1-23.

[3] M. Zhang, Y. Zhang, X. Cheng, An enhanced coupling PD with sliding mode control method for underactuated double-pendulum overhead crane systems, Int. J. Control. Autom. Syst. 17 (2019) 1579-1588.

[4] N. Sun, T. Yang, Y. Fang, Y. Wu, H. Chen, Transportation control of double-pendulum 
cranes with a nonlinear quasi-PID scheme: Design and experiments, IEEE Trans. Syst. Man, Cybern. Syst. 49 (2019) 1408-1418.

[5] L. Ramli, Z. Mohamed, M.O. Efe, I.M. Lazim, H.I. Jaafar, Efficient swing control of an overhead crane with simultaneous payload hoisting and external disturbances, Mech. Syst. Signal. Process. 135 (2020) 106326.

[6] H. Yavuz, S. Beller, An intelligent serial connected hybrid control method for gantry cranes, Mech. Syst. Signal Process. 146 (2021) 107011.

[7] B. Lu, Y. Fang, N. Sun, Enhanced-coupling adaptive control for double-pendulum overhead cranes with payload hoisting and lowering, Automatica. 101 (2019) 241-251.

[8] Y. Wu, N. Sun, H. Chen, Y. Fang, Adaptive output feedback control for 5-DOF varyingcable-length tower cranes with cargo mass estimation, IEEE Trans. Ind. Informatics. (2020), https://doi.org/10.1109/TII.2020.3006179, In Press.

[9] T. Yang, N. Sun, H. Chen, Y. Fang, Observer-based nonlinear control for tower cranes suffering from uncertain friction and actuator constraints with experimental verification, IEEE Trans. Ind. Electron. (2020), https://doi.org/10.1109/TIE.2020.2992972, In Press.

[10] X. Wu, K. Xu, X. He, Disturbance-observer-based nonlinear control for overhead cranes subject to uncertain disturbances, Mech. Syst. Signal Process. 139 (2020) 106631.

[11] S. Zhang, X. He, H. Zhu, Q. Chen, Y. Feng, Partially saturated coupled-dissipation control for underactuated overhead cranes, Mech. Syst. Signal Process. 136 (2020) 106449 .

[12] A.M. Abdullahi, Z. Mohamed, H. Selamat, H.R. Pota, M.S. Zainal Abidin, S.M. Fasih, Efficient control of a 3D overhead crane with simultaneous payload hoisting and wind disturbance: design, simulation and experiment, Mech. Syst. Signal Process. 145 (2020) 106893.

[13] Q. Wu, X. Wang, L. Hua and M. Xia, Dynamic analysis and time optimal anti-swing control of double pendulum bridge crane with distributed mass beams, Mech. Syst. Signal Process. 144 (2020) 106968.

[14] I.A. Martin, R.A. Irani, A generalized approach to anti-sway control for shipboard cranes, Mech. Syst. Signal Process. 148 (2021) 107168.

[15] L. Ramli, I.M. Lazim, H.I. Jaafar, Z. Mohamed, Modelling and fuzzy logic control of an underactuated tower crane system, Applications of Modelling and Simulation. 4 (2020) $1-11$.

[16] H.I. Jaafar, Z. Mohamed, N.A. Mohd Subha, A.R. Husain, F.S. Ismail, L. Ramli, M.O. Tokhi, M.A. Shamsudin, Efficient control of a nonlinear double-pendulum overhead 
crane with sensorless payload motion using an improved PSO-tuned PID controller, J. Vib. Control. 25 (2019) 907-921.

[17] N. Sun, Y. Wu, Y. Fang, H. Chen, Nonlinear antiswing control for crane systems with double-pendulum swing effects and uncertain parameters: Design and experiments, IEEE Trans. Autom. Sci. Eng. 15 (2018) 1413-1422.

[18] H. Ouyang, J. Hu, G. Zhang, L. Mei, X. Deng, Sliding-mode-based trajectory tracking and load sway suppression control for double-pendulum overhead cranes, IEEE Access. 7 (2019) 4371-4379.

[19] D. Qian, S. Tong, S. Lee, Fuzzy-logic-based control of payloads subjected to doublependulum motion in overhead cranes, Autom. Constr. 65 (2016) 133-143.

[20] H. Ouyang, J. Hu, G. Zhang, L. Mei, X. Deng, Decoupled linear model and S-shaped curve motion trajectory for load sway reduction control in overhead cranes with doublependulum effect, Proc. Inst. Mech. Eng. Part C J. Mech. Eng. Sci. 233 (2019) 3678-3689.

[21] M. Giacomelli, F. Padula, L. Simoni, A. Visioli, Simplified input-output inversion control of a double pendulum overhead crane for residual oscillations reduction, Mechatronics. 56 (2018) 37-47.

[22] W. Singhose, Command shaping for flexible systems: A review of the first 50 years, Int. J. Precis. Eng. Manuf. 10 (2009) 153-168.

[23] K.-S. Hong, U.H. Shah, Dynamics and control of industrial cranes, 1st ed., Springer Nature Singapore Pte Ltd., Singapore, 2019.

[24] M. Kenison, W. Singhose, Input shaper design for double-pendulum planar gantry cranes, in: Proc. IEEE Int. Conf. Control Appl., Hawaii, USA, 1999: pp. 539-544.

[25] W. Singhose, J. Lawrence, K. Sorensen, D. Kim, Applications and educational uses of crane oscillation control, FME Trans. 34 (2006) 175-183.

[26] K.C.C. Peng, W. Singhose, S.S. Gurleyuk, Initial investigations of hand-motion crane control with double-pendulum payloads, in: Am. Control Conf., Montréal, Canada, 2012: pp. 6270-6275.

[27] K.A. Alhazza, Z.N. Masoud, Waveform command shaping control of multimode systems, J. Sound Vib. 363 (2016) 126-140.

[28] Z.N. Masoud, K.A. Alhazza, A smooth multimode waveform command shaping control with selectable command length, J. Sound Vib. 397 (2017) 1-16.

[29] X. Xie, J. Huang, Z. Liang, Vibration reduction for flexible systems by command smoothing, Mech. Syst. Signal Process. 29 (2013) 461-470.

[30] J. Huang, X. Xie, Z. Liang, Control of bridge cranes with distributed-mass payload 
dynamics, IEEE/ASME Trans. Mechatronics. 20 (2015) 481-486.

[31] R. Tang, J. Huang, Control of bridge cranes with distributed-mass payloads under windy conditions, Mech. Syst. Signal Process. 72-73 (2016) 409-419.

[32] M.J. Maghsoudi, L. Ramli, S. Sudin, Z. Mohamed, A.R. Husain, H. Wahid, Improved unity magnitude input shaping scheme for sway control of an underactuated 3D overhead crane with hoisting, Mech. Syst. Signal Process. 123 (2019) 466-482.

[33] S.M. Fasih, Z. Mohamed, A.R. Husain, L. Ramli, A. M. Abdullahi, W. Anjum, Payload swing control of a tower crane using a neural network-based input shaper, Measurement and Control. (2020), https://doi.org/10.1177/0020294020920895, In Press.

[34] H.I. Jaafar, Z. Mohamed, M.A. Shamsudin, N.A. Mohd Subha, L. Ramli, A.M. Abdullahi, Model reference command shaping for vibration control of multimode flexible systems with application to a double-pendulum overhead crane, Mech. Syst. Signal Process. 115 (2019) 677-695.

[35] J. Vaughan, E. Maleki, W. Singhose, Advantages of using command shaping over feedback for crane control, in: Am. Control Conf., MD, USA, 2010: pp. 2308-2313.

[36] M.A. Ahmad, R.M.T.R. Ismail, M.S. Ramli, N. Hambali, Investigations of NCTF with input shaping for sway control of a double-pendulum-type overhead crane, in: Proc. 2nd Int. Conf. Adv. Comput. Control, Shenyang, China, 2010: pp. 456-461.

[37] M.Z.M. Tumari, M.S. Saealal, K.H. Ghazali, M.R. Ghazali, Comparative study of input shaping with different polarities and modes selection in hybrid control schemes of a DPTOC system, Int. J. Syst. Signal Control Eng. Appl. 5 (2012) 5-13.

[38] D. Fujioka, W. Singhose, Optimized input-shaped model reference control on doublependulum system, J. Dyn. Syst. Meas. Control. 140 (2018) 1-9.

[39] R. Mar, A. Goyal, V. Nguyen, T. Yang, W. Singhose, Combined input shaping and feedback control for double-pendulum systems, Mech. Syst. Signal Process. 85 (2017) $267-277$.

[40] K.A. Alhazza, A.M. Hasan, K.A. Alghanim, Z.N. Masoud, An iterative learning control technique for point-to-point maneuvers applied on an overhead crane, Shock Vib. 2014 (2014) 1-11.

[41] M.J. Maghsoudi, Z. Mohamed, A.R. Husain, M.O. Tokhi, An optimal performance control scheme for a 3D crane, Mech. Syst. Signal Process. 66-67 (2016) 756-768.

[42] J. Smoczek, J. Szpytko, Particle swarm optimzation-based multivariable generalized predictive control for an overhead crane, IEEE/ASME Trans. Mechatronics. 22(1) (2017) $258-268$. 
[43] H. Liu, W. Cheng, Using the bezier curve and particle swarm optimization in trajectory planning for overhead cranes to surpress the payload residual swing, Math. Probl. Eng. 2018 (2018) 1-13.

[44] A.P. Piotrowski, J.J. Napiorkowski, A.E. Piotrowska, Population size in particle swarm optimization, Swarm and Evolutionary Computation. 58 (2020) 100718.

[45] J. Kennedy, R. Eberhart, Particle swarm optimization, in: Proc. IEEE Int. Conf. Neural Networks, Perth, Australia, 1995, pp. 1942-1948.

[46] L. Ramli, Z. Mohamed, H.I. Jaafar, A neural network-based input shaping for swing suppression of an overhead crane under payload hoisting and mass variations, Mech. Syst. Signal Process. 107 (2018) 484-501.

[47] N. Sun, J. Zhang, X. Xin, T. Yang, Y. Fang, Nonlinear output feedback control of flexible rope crane systems with state constraints, IEEE Access. 7 (2019) 136193-136202. 